\title{
Curvature of BEOL Cantilevers in CMOS-MEMS Processes
}

\author{
Juan Valle, Daniel Fernández, Jordi Madrenas and Laura Barrachina
}

\begin{abstract}
This article presents the curvature characterization results of released back-end-of-line (BEOL) $5 \mu \mathrm{m}$-wide cantilevers for two different $0.18 \mu \mathrm{m} 1 \mathrm{P} 6 \mathrm{M}$ complementary metal-oxide semiconductor microelectromechanical systems (CMOS-MEMS) processes. Results from different runs and lots from each foundry are presented. The methodology and accuracy of the characterization approach, based on optical measurements of test cantilever curvature are also discussed. Special emphasis is given to the curvature average and variability as a function of the number of stacked layers. Analythical equations for modeling the bending behavior of stacked cantilevers as a function of the tungsten (W) vias that join the metal layers are presented. In addition, the effect of various post-processing conditions and design techniques on the curvature of both single and stacked cantilevers is analyzed. In particular, surpassing certain timedependent temperature stress conditions after release lead to curvature shifts larger than one order of magnitude. Also, the W via design was found to strongly affect the curvature of the test cantilevers.
\end{abstract}

Index Terms-CMOS-MEMS, Bending Stiffness, Curvature, Stacks, Temperature, Test Cantilevers

\section{INTRODUCTION}

M ONOLITHIC integration of CMOS electronics and MEMS devices can potentially reduce fabrication costs and achieve smaller size and lower parasitics than other integration approaches. One way to achieve CMOS-MEMS monolithic integration is by using the back-end-of-line (BEOL) layers of the CMOS process as structural layers for the MEMS device (CMOS-MEMS micromachining) [1, 2].

One of the main concerns of CMOS-MEMS processes is the curvature of the released BEOL structural layers [3-5], which is caused by the residual stresses and thermal coefficient mismatches of the different layers that form each BEOL layer. These are not tightly monitored or controlled by the CMOS foundries [6] given that their effects mostly arise after a release step only necessary for MEMS fabrication and, therefore, do not pose a strong concern for the reliability of standard CMOS circuits. Unfortunately, this curvature is typically large and, most importantly, its repeatability is poor as we shall see in section V. These limitations impose device size limits and

J. Valle and J. Madrenas are with the Department of Electronic Engineering, Universitat Politècnica de Catalunya, Jordi Girona 1 i 3, Edifici C4, 08034 Barcelona, Spain (e-mail: juan.valle.fraga@gmail.com, jordi.madrenas@upc.edu)

D. Fernández is with Nanusens, Av. del Parc Tecnològic 3, CENT Parc Tecnològic del Vallès, 08290 Cerdanyola del Vallès, Spain (e-mail: daniel.fernandez@nanusens.com)

L. Barrachina is with Owlstone, 127 Cambridge Science Park, Milton Road, Cambridge CB4 0GD, United Kingdom (e-mail: laura.barrachina@owlstone.co.uk). therefore reduce the range of valid MEMS designs compared to other MEMS processes $[4,7,8]$. The variability of process and design parameters is particularly important in the case of commercial devices, which need to achieve the required levels of performance in the full range of process variability. The curvature of the BEOL layers plays a very important role in this sense and, although there are plenty of works dealing with curvature and residual stresses [5, 9-14], no large studies regarding the curvature of the BEOL metal layers of CMOSMEMS processes are available in the literature.

A well-known method to achieve flatter CMOS-MEMS structures is layer stacking or composite cantilevers $[2,5$, $9,15]$, but statistical data, curvature variability and mathematical description of the mechanical bending behaviour is not presented in these studies. In addition, although analytical modeling of multilayered structures was presented in [16], analytical formulas for modeling the bending behavior when the layers are joined with vias is not found in the literature. Thus, curvature characterization of single and composite cantilevers of CMOS-MEMS processes joined with tungsten (W) vias is one of the main objectives of this paper.

In this work we analyze the limitations of test cantilever curvature measurement and the effect on curvature of different processing conditions, like etch time or temperature history, and design variations, such as $\mathrm{W}$ via design or layer stacking. A large database is constructed from all the analyzed samples from two different CMOS-MEMS processes. We will begin by describing the CMOS-MEMS fabrication process in section II. Then, the general relationship between curvature and residual stress is briefly introduced in section III. The characterization methodology employed in this paper and its limitations are explained in section IV, where analythical equations that describe the mechanical bending behaviour and curvature of metal stacks joined with $\mathrm{W}$ vias are also presented. The results are discussed in section $\mathrm{V}$, where the effects on curvature of thickness variations, several processing conditions and design variations are discussed. In particular, the effects on final curvature of past temperature stress and of different via design that join the BEOL metal layers are studied in detail.

\section{CMOS-MEMS FABRICATION PROCESS}

The CMOS process flow can be divided in two parts: the front-end-of-line (FEOL), where the active devices, mainly transistors, are built; and the BEOL, where the metal interconnecting layers are deposited. The CMOS-MEMS devices studied in this article are built with the BEOL materials. Initially, as fabricated by the CMOS foundry, the MEMS structures are surrounded by the BEOL inter-metal dielectric 


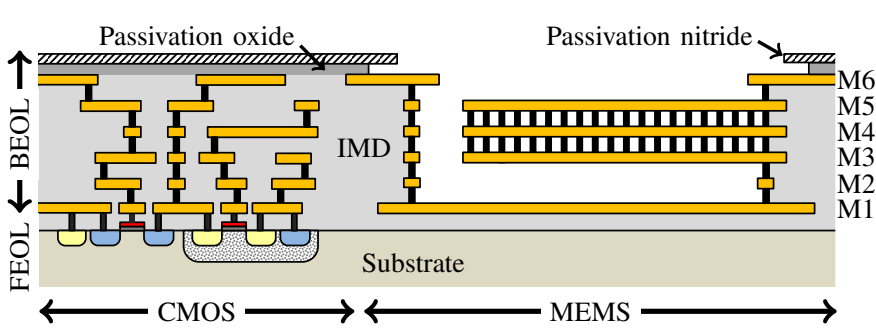

Figure 1: Cross section of a cantilever made of a stack of layers M3, M4, M5 and the $\mathrm{W}$ vias that join them (in black) after the vHF release process in a typical $0.18 \mu \mathrm{m} 1 \mathrm{P} 6 \mathrm{M}$ CMOS process. The vHF is masked by the passivation nitride layer (dashed) that only allows IMD oxide etching in the MEMS area. Note the cavity around the cantilever used to confine the etching.

(IMD) oxide. The sacrificial IMD oxide is etched away with a vapor HF (vHF) process [17] that does not etch the BEOL metals, releasing the MEMS structures as in figure 1 , and providing them with their functionality, i.e., freedom to move or others. After the vHF release some impurities are left from the etching reaction [17], which can be removed with a baking step, which typically consists on rising the temperature to $250{ }^{\circ} \mathrm{C}$ approximately during one minute. The BEOL materials may be combined in several ways when creating the MEMS structure. For example, a single layer of metal may be used, or several layers of metal may be stacked with the aid of vias as shown in figure 1. Other ways may be possible, depending on the specific options of the CMOS-MEMS process. Finally, the MEMS devices are sealed, diced and packaged [18].

While $0.18 \mu \mathrm{m}$ CMOS technologies are currently the sweet spot (lowest overall cost) for mixed-signal applications, a large number of BEOL metal layers improves MEMS design flexibility. For these two reasons, and for widening the generality of this work, two 1P6M 0.18 $\mu \mathrm{m}$ CMOS processes were used: one from Global Foundries (GF) and another from LFoundry (LF). In terms of MEMS design, the main difference between both processes is the thickness of the BEOL metal layers and the gaps between them, being greater for the GF process. Typically, thicker layers lead to reduced curvatures, greater stiffness and larger minimum separation between features. Which process is the best suited will be determined by the requirements of the MEMS application.

The BEOL metal layers of the two CMOS processes used have the same base materials (Al-based alloy, Ti and TiN). The metal layers are generally labeled according to the deposition order (metal 1 is the bottom metal layer and metal 6 is the top layer). The thickness of the BEOL layers was measured with focused-ion-beam (FIB) cuts, which cannot distinguish between $\mathrm{Ti}$ and TiN layers, yielding the results of table I, where the error is never lower than $10 \%$ to account for the typical BEOL thickness variations in CMOS processes.

\section{WHY THIN FILMS CURVE}

After release, the initially flat cantilevers of a CMOSMEMS process curl up or down depending on their initial residual stresses. Residual stress may arise from intrinsic stress gradients originated during the formation of the cantilever, or from thermal coefficient mismatches (different materials, same material but slightly different deposition conditions, etc.).

\begin{tabular}{|c|c|c|c|c|c|}
\hline \multicolumn{4}{|c|}{ Metal cross section } & $\mathrm{LF}$ & GF \\
\hline \multicolumn{2}{|r|}{ Ti/TiN } & \multirow{2}{*}{$\mathrm{a}$} & M1-M5 t (nm) & $400 \pm 40$ & $570 \pm 57$ \\
\hline & \multirow{3}{*}{ Al-based } & & M1-M5 a (nm) & $40 \pm 15$ & $60 \pm 15$ \\
\hline $\mathrm{t}$ & & \multirow[t]{2}{*}{$\mathrm{b}$} & M1-M5 b (nm) & $320 \pm 32$ & $440 \pm 44$ \\
\hline \multirow{3}{*}{$\downarrow$} & & & M1-M5 c (nm) & $40 \pm 15$ & $70 \pm 15$ \\
\hline & Ti/TiN & $\mathrm{c}$ & M6 t (nm) & $900 \pm 90$ & $900 \pm 90$ \\
\hline & & & en metals (nm) & $500 \pm 50$ & $900 \pm 90$ \\
\hline
\end{tabular}

Table I: MEAsured BEOL Thicknesses.

The average residual stress creates an expansion or contraction force $F$ that will lead to the corresponding cantilever expansion or contraction when it is not constrained. Likewise, nonuniform axial residual stress (usually referred to as residual stress gradient) creates a bending moment $M$, which will curl the cantilever when it is not constrained. Mechanical equilibrium relates the expansion force $F$ and the bending moment $M$ caused by the initial residual stress distribution $\sigma$ as described by the following equations [19]:

$$
\begin{aligned}
& F=-\int_{A} \sigma \mathrm{d} A=0 \\
& M=\int_{A} \sigma z \mathrm{~d} A=0
\end{aligned}
$$

where $A$ is the cross-sectional area and $z$ the distance to the neutral axis (the axis at which strain and stress are zero when the beam is subjected to bending when no initial residual stress is present.)

The expansion force $F$ produces a length increment $\Delta L$ equal to [19]:

$$
\Delta L=\frac{F L}{E A}
$$

where $L$ is the cantilever length and $E$ the Young's Modulus.

Also, the bending moment $M$ produces a curvature $K$, which are related by the so-called moment-curvature equation [19]:

$$
K=\frac{M}{E I}
$$

where $E$ is the Young Modulus and $I$ the second moment of area of the beam's cross section. The product EI is usually called flexural rigidity or bending stiffness, and it is proportional to the spring constant of beams under bending when the residual stress effect is negligible.

\section{CURVATURE ChARACTERIZATION METHOD}

\section{A. Measurement Setup}

The curvature characterization was performed by measuring the curved profile of test cantilevers uniformly distributed over the wafers in order to extract with-in-wafer (WIW) uniformity. The topography of the test cantilevers after release (see figure 2a) was measured optically with a Reflection Digital Holographic Microscope (DHM) from Lyncée-Tec [20]. These type of microscopes record holograms like in figure $2 b$, which contain both the intensity and the phase of the incident 
light, allowing digital focusing during post-processing and precise curvature measurements. Due to surface roughness, residues and image quality our vertical resolution was around $10 \mathrm{~nm}$, while the horizontal resolution was around $1.5 \mu \mathrm{m}$, which proved enough for our purposes. All the holograms were taken automatically at wafer level with the aid of a Cascade probe station 12000b assembled with Lyncée-tec's DHM R1000 allowing us to perform automatic on wafer measurements. All measurements were performed in a clean room controlled environment at $22{ }^{\circ} \mathrm{C}$ and $45 \%$ of relative humidity. The profiles of the test cantilevers were extracted from their corresponding holograms automatically with Matlab using an image recognition algorithm. Then, the extracted profiles were fitted to a circumference, obtaining, this way, the curvature radius $R$ and the curvature $K=1 / R$ of the cantilevers. The fit was generally very good, as expected for cantilevers with uniform curvature along their length. How circular the cantilever profile is was quantified with the circularity parameter, defined as the distance between the two closest concentric circles that enclose the cantilever profile, and defined as:

$$
\text { Circularity }=\min _{\forall\left(x_{c}, y_{c}\right)}\left(R_{a}-R_{b}\right)
$$

where $\left(x_{c}, y_{c}\right)$ are the center coordinates of two concentric circles with radii $R_{a}$ and $R_{b}$, which satisfy:

$$
y_{c}+\sqrt{R_{a}^{2}-\left(x-x_{c}\right)^{2}} \leq y(x) \leq y_{c}+\sqrt{R_{b}^{2}-\left(x-x_{c}\right)^{2}}
$$

for $0 \leq x \leq L$, and where $y(x)$ is the cantilever profile.

The smaller the circularity, the better the cantilever follows a circular shape. In practice, it is not zero due to several factors, like surface roughness, measurement noise and curvature nonuniformity along the length of the cantilever. In order to determine if curvature is the most important contributor to cantilever profile, the measured circularity values will be compared in section V-B with the theoretical tip displacement $\left(z_{t i p}\right)$ due to curvature of the measured cantilevers, which is:

$$
z_{\text {tip }}=\frac{2}{K} \sin ^{2}\left(\frac{K L}{2}\right) \approx \frac{K L^{2}}{2} \text { for } K L \rightarrow 0
$$

where $K$ and $L$ are the cantilever curvature and length, respectively. A zero initial slope at the anchor point is assumed.

\section{B. Test Structure Design and Modeling}

Clamped-free cantilevers were used for monitoring the BEOL metal layers curvature after vHF release. Each BEOL metal layer (M2, M3, M4, M5 and M6) had its corresponding test cantilevers, which were grouped in arrays of 7 cantilevers (see figure 2a), each of a different length. Several lengths are needed in order to deal with very different curvature values. The cantilever width needs to be large enough to be compatible with the horizontal resolution of the measuring optical system and to avoid low aspect ratio effects that can take place when the width is comparable to the thickness of the layer and which can influence the curvature of the cantilever $[4,21]$. The width also needs to be thin enough to avoid undesirable anchor and

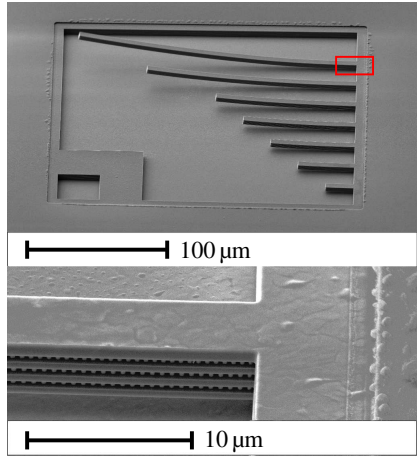

(a) SEM image (top) and zoomed region at the anchor (bottom).

Figure 2: Test Cantilevers of metals M3456 after release. Taken from Half of Wafer 2, Lot 1, Run 1, LFoundry.

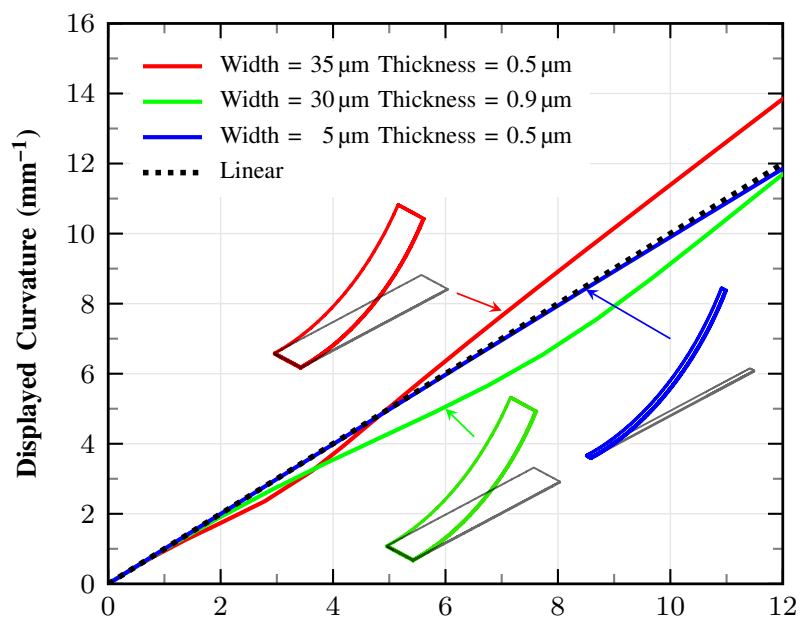

Equivalent Applied Strain Gradient / Curvature $\left(\mathbf{m m}^{-1}\right)$

Figure 3: ANSYS simulation results showing the non-linear relationship between applied gradient strain and curvature for three clamped-free cantilevers of different widths and thicknesses (Length $=200 \mu \mathrm{m}$, Poisson's ratio $=0.3$ ). Non-linearities are significantly more pronounced for the wider and thinner cantilevers, but are negligible below $1 \mathrm{~mm}^{-1}$.

non-linear effects (see figure 3) which would prevent making a readily linear correspondence between curvature and gradient stress or strain. The width was chosen to be $5 \mu \mathrm{m}$, which complied with both requirements. Typically, no appreciable curvature dependence on cantilever width was observed for moderate curvature levels and cantilevers ranging from $5 \mu \mathrm{m}$ to $30 \mu \mathrm{m}$, as in the case shown in figure 4 . The used lengths were $20 \mu \mathrm{m}, 40 \mu \mathrm{m}, 60 \mu \mathrm{m}, 80 \mu \mathrm{m}, 100 \mu \mathrm{m}, 150 \mu \mathrm{m}$ and $200 \mu \mathrm{m}$.

In our experience, the curvature along $\mathrm{x}$ and $\mathrm{y}$ directions was the same when the structure was free to curve and the curvature was small enough to avoid geometrical nonlinearities, so curvature characterization along more than one direction would be redundant.

Also, CMOS-MEMS cantilevers (and other structures) may be composed of several layers joined with $\mathrm{W}$ vias, here referred to as stacked cantilevers. For example, M234 is the short for a stack made of metal layers 2, 3 and 4. Metal stacks are very useful for reducing the curvature [2] at the expense of 


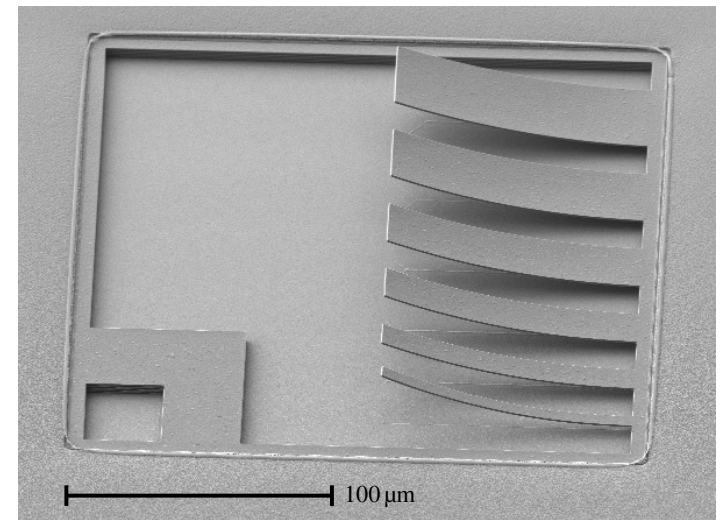

Figure 4: SEM image showing released $100 \mu \mathrm{m}$-long M6 cantilevers of different widths $(5,10,15,20,25$ and $30 \mu \mathrm{m})$. Note that their curvature $(\approx$ $3 \mathrm{~mm}^{-1}$ ) does not appreciably depend on the cantilever width. Taken from Half of Wafer 2, Lot1, Run 1, LFoundry.

higher bending stiffness as described by equation 4 . Statistical curvature data of all the possible stacks is, therefore, very interesting, so the corresponding test cantilevers were included in all analyzed wafers. These are different from the stacks used in other CMOS-MEMS processes as in this study only metals are used, and there is no oxide surrounding the metal layers as in $[3,9,22-24]$, which is not possible in our case due to our release process characteristics. The typical stacks used in this work are metal cantilevers joined by $\mathrm{W}$ vias distributed in a rectangular array as they are commonly used in CMOS designs. However, other non-standard via designs were tested and are described in section V-J.

Using equations 2 and 4, the bending stiffness of the stacks $\left(E I_{\text {stack }}\right)$ as a function of the number of stacked layers was calculated analytically and is shown in table II. The coefficient $\alpha$ accounts for the type of $\mathrm{W}$ via design used for joining the metal layers, which has to be determined numerically or empirically. For a solid W layer between the metal layers $\alpha=1$. Interestingly, for the rectangular $\mathrm{W}$ via array used in this work, numerical simulations show and experiments indicate (see section $\mathrm{V}-\mathrm{J}$ ) that the $\mathrm{W}$ vias do not contribute to $E I_{\text {stack }}$, in which case $\alpha \sim 0$. This, and other $\mathrm{W}$ via designs for which $1>\alpha>0$ are further discussed in section V-J. In addition, $E_{L F}, I_{L F}, E_{G F}$ and $I_{G F}$ stand for the effective Young's modulus and inertia moment of a single metal layer of LF and GF processes; $t$ and $w$ are the considered thickness and width, respectively. Coefficient $\gamma$ is equal to the ratio $E_{W} / E$, where $E_{W}$ and $E$ are the Young's modulus of the via material and the effective Young Modulus of a single metal layer $\left(E_{L F}\right.$ or $\left.E_{G F}\right)$. Columns 3 and 4 show the EI values for LF and GF stacks, and are expressed in two different ways: the top line compares $E I_{\text {stack }}$ to the $E I$ of a single layer, and the second line is useful for modeling the stack as a solid beam with an equivalent Young's Modulus (0.93E for a LF 2-metal stack, for example).

The deformation of a 2-metal stack from GF due to electrostatic actuation was used to calculate the effective Young modulus of the stack by comparison with FE simulations of a solid beam, which yielded $E_{2 \text { metals }}=180 \pm 20 \mathrm{GPa}$.
Applying the corresponding coefficient from table II (0.92), the effective Young's modulus of a single metal layer from GF was calculated, yielding $E_{G F}=196 \pm 22 \mathrm{GPa}$.

Using equation 4 , the curvature of a stack $\left(K_{\text {stack }}\right)$ can be estimated from the curvature of the single metals $\left(K_{i}\right)$, their average residual stress $\left(\sigma_{i}\right)$ and the bending moment produced by the vias $\left(M_{W j}\right)$, as described by the following equation:

$$
K_{\text {stack }}=\sum_{i} K_{i} \frac{E I_{i}}{E I_{\text {stack }}}+\sum_{i} \frac{\sigma_{i} t_{i} w z_{i}}{E I_{\text {stack }}}+\sum_{j} \frac{M_{W j}}{E I_{\text {stack }}}
$$

where, $E I_{i}$ and $E I_{\text {stack }}$ are the bending stiffness of the ith layer and the stack, respectively, defined in table II; $w$ is the cantilever width, and $t_{i}$ and $z_{i}$ are the thickness and $\mathrm{z}$ position of the ith layer center with respect to the neutral axis, respectively.

\section{Accuracy and Limitations}

Although curvature displayed by the cantilevers is measured optically, not all cantilever lengths are suitable for curvature monitoring: short cantilevers may be too flat (high curvature measurement error) and long ones too curved (touching the substrate or exceeding maximum slope measurable by the DHM). These limitations are discussed in the following.

1) Condition 1: Avoid touching the substrate. In order to avoid touching the substrate, the length $L$ of a test cantilever must be:

$$
L<\frac{1}{|K|} \arcsin \left(|K| \sqrt{\left(\frac{2}{|K|}-g\right) g}\right)
$$

for an initial gap $g$ between the test cantilever and the substrate, and a negative curvature $K$.

Since typically the radius of curvature is much larger than the distance to the substrate $(1 / K \gg g)$, the previous condition may be simplified to:

$$
L<\sqrt{\frac{2 g}{|K|}}
$$

For example, figure 5 shows that cantilevers larger than $60 \mu \mathrm{m}$ touch the $\mathrm{m} 1$ substrate, so $60 \mu \mathrm{m}$-long cantilevers were used in that case.

2) Condition 2: Maximum slope. Holograms taken by the DHM are composed of fringes (see figure 2b) that correspond to different z-positions of the analyzed surface. Each fringe represents a vertical range of $341.25 \mathrm{~nm}$. Larger slopes lead to narrower fringes that may be beyond the horizontal resolution of the measurement setup. Therefore, when a given slope is reached the cantilever profile cannot be measured as shown in figure 6. Experimental data showed that, in our setup, the maximum measurable slope $\left(y_{m}^{\prime}\right)$ was around 0.2 . This imposes a relationship between the curvature and the maximum measurable length of the test cantilevers, which is:

$$
L<\frac{1}{|K|}\left(\theta_{m}-\theta_{i}\right) \approx \frac{0.1}{|K|}
$$




\begin{tabular}{|c|c|c|c|}
\hline \multirow{2}{*}{$\begin{array}{l}\text { Number } \\
\text { of layers }\end{array}$} & \multirow{2}{*}{$\begin{array}{l}\text { Stack Bending Stiffness }\left(E I_{\text {stack }}\right) \\
\text { Analytic Formula }\end{array}$} & \multicolumn{2}{|c|}{$E I_{\text {stack }}(\alpha=0)$} \\
\hline & & $\operatorname{LF}\left(\frac{g}{t} \sim 1.25\right)$ & $\mathrm{GF}\left(\frac{g}{t} \sim 1.58\right)$ \\
\hline $1 \Longrightarrow$ & $E I=E \frac{1}{12} w t^{3}$ & $\begin{array}{c}1 \cdot E_{L F} I_{L F} \\
I_{L F}=I(t=0.40 \mu \mathrm{m})\end{array}$ & $\begin{array}{c}1 \cdot E_{G F} I_{G F} \\
I_{G F}=I(t=0.57 \mu \mathrm{m})\end{array}$ \\
\hline $2 \overline{\mathbf{I n}}$ & $E I\left[8+12\left(\frac{g}{t}\right)+6\left(\frac{g}{t}\right)^{2}+\alpha \gamma\left(\frac{g}{t}\right)^{3}\right]$ & $\begin{array}{c}32 \cdot E_{L F} I_{L F}= \\
0.93 \cdot E_{L F} I(t=1.30 \mu \mathrm{m})\end{array}$ & $\begin{array}{c}42 \cdot E_{G F} I_{G F}= \\
0.92 \cdot E_{L F} I(t=2.04 \mu \mathrm{m})\end{array}$ \\
\hline$3 \longdiv { \overline { 1 0 } }$ & $E I\left\{27+48\left(\frac{g}{t}\right)+24\left(\frac{g}{t}\right)^{2}+\alpha \gamma\left[6\left(\frac{g}{t}\right)+12\left(\frac{g}{t}\right)^{2}+8\left(\frac{g}{t}\right)^{3}\right]\right\}$ & $\begin{array}{c}125 \cdot E_{L F} I_{L F}= \\
0.75 \cdot E_{L F} I(t=2.20 \mu \mathrm{m})\end{array}$ & $\begin{array}{c}163 \cdot E_{G F} I_{G F}= \\
0.70 \cdot E_{L F} I(t=3.51 \mu \mathrm{m})\end{array}$ \\
\hline 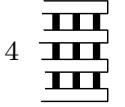 & $E I\left\{64+120\left(\frac{g}{t}\right)+60\left(\frac{g}{t}\right)^{2}+\alpha \gamma\left[24\left(\frac{g}{t}\right)+48\left(\frac{g}{t}\right)^{2}+27\left(\frac{g}{t}\right)^{3}\right]\right\}$ & $\begin{array}{c}308 \cdot E_{L F} I_{L F}= \\
0.66 \cdot E_{L F} I(t=3.10 \mu \mathrm{m})\end{array}$ & $\begin{array}{c}403 \cdot E_{G F} I_{G F}= \\
0.60 \cdot E_{L F} I(t=4.98 \mu \mathrm{m})\end{array}$ \\
\hline
\end{tabular}

Table II: Bending Stiffness (EI) AS a Function of Number of Layers Stacked.

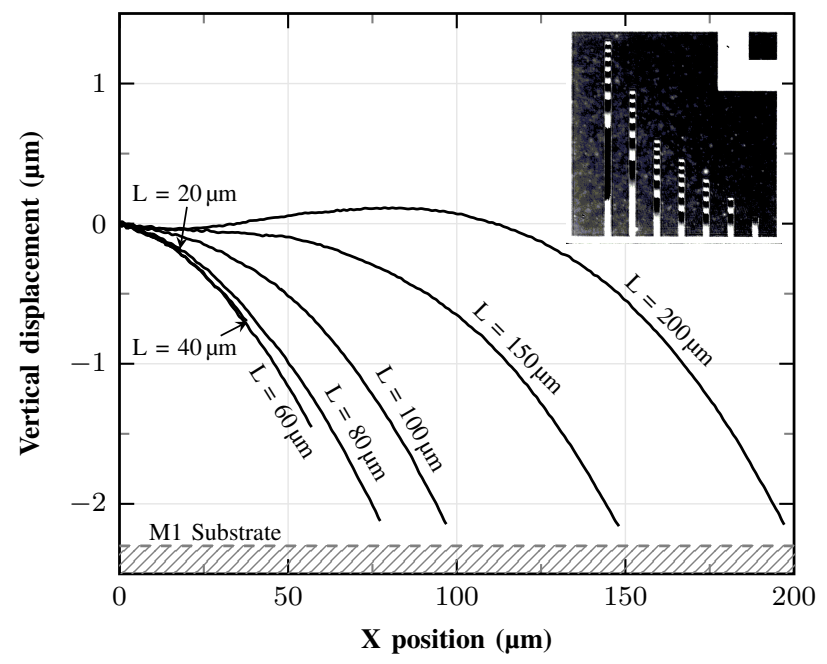

Figure 5: Profiles of the 7 cantilevers from a m45 test structure curved downwards (negative curvature). The longest ones are touching the M1 substrate, represented with a dashed region. Note that the profile near the tip is not measured to avoid border effects. Taken from Quarter of Wafer 2, Lot1, Run 2, LFoundry.

where

$$
\begin{gathered}
\theta_{m}=\left.\arcsin \left(\frac{y_{m}^{\prime}}{\sqrt{1+\left(y_{m}^{\prime}\right)^{2}}}\right)\right|_{y_{m}^{\prime}=0.2} \approx 0.2 \\
\theta_{i}=\left.\arcsin \left(\frac{y_{i}^{\prime}}{\sqrt{1+\left(y_{i}^{\prime}\right)^{2}}}\right)\right|_{y_{i}^{\prime}=0.1} \approx 0.1
\end{gathered}
$$

and where $y_{i}^{\prime}$ is the slope of the cantilever at the anchoring point. In our case, initial slopes smaller than 0.1 conform the vast majority of the analyzed test cantilevers. Values derived from equation 11 for each of the cantilever lengths used in this article are shown in table III.

3) Condition 3: Accuracy. Small curvatures cannot be measured accurately with short cantilevers as they remain very flat and vertical deformations due to the curvature are smaller or comparable to several error sources (surface roughness, imperfections and small residues, measurement noise, misfocusing, etc). In order to quantify the measurement error, several known curvature levels were measured with cantilevers

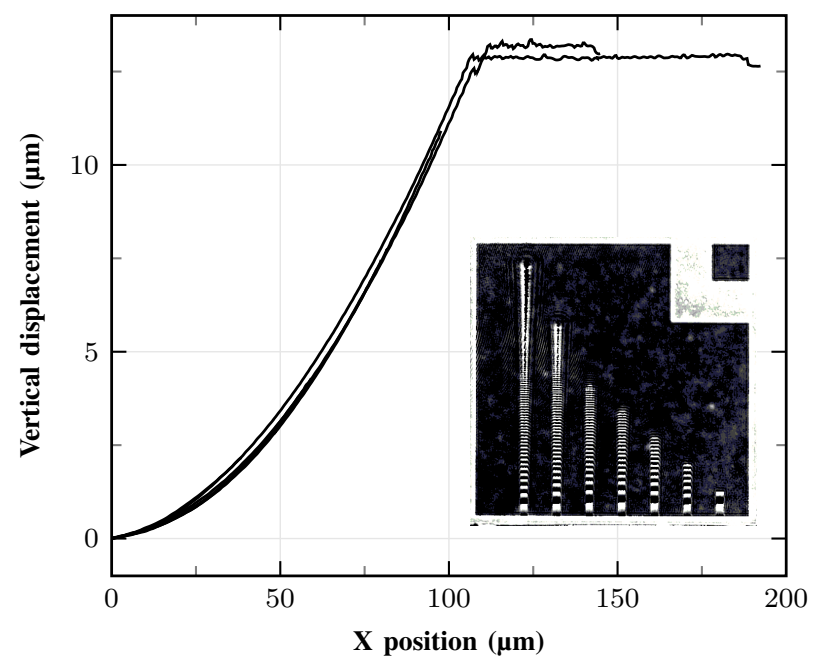

Figure 6: Profiles of the 7 cantilevers from a $\mathrm{m} 4$ test structure. The longest cantilevers surpass the maximum allowable slope at an approximate distance of $110 \mu \mathrm{m}$ from the anchoring point. Hologram of the plotted profiles is at the bottom right corner.

\begin{tabular}{ccc}
\hline $\begin{array}{c}\text { Cantilever length } \\
(\mu \mathrm{m})\end{array}$ & $\begin{array}{c}\text { Curvature limit } \\
\left(\mathrm{mm}^{-1}\right)\end{array}$ & $\begin{array}{c}\text { Curvature error } \\
(1 / \mathrm{mm})\end{array}$ \\
\hline 200 & 0.51 & 0.0021 \\
150 & 0.69 & 0.0038 \\
100 & 1.05 & 0.0089 \\
80 & 1.33 & 0.0142 \\
60 & 1.82 & 0.0264 \\
40 & 2.86 & 0.0653 \\
20 & 6.67 & 0.3556 \\
\hline
\end{tabular}

Table III: MEAsurement Limitations IMPOSED BY THE LENGTH OF THE TEST CANTILEVERS

of different lengths. For each cantilever, the measurement was repeated several times, yielding slightly different values in each observation: mean curvature $\left(K_{\text {mean }}\right)$ and standard deviation $\left(K_{\text {dev }}\right)$ values were obtained. Measured variability increased substantially for shorter test cantilevers. Also, $K_{\text {mean }}$ did not match perfectly the curvature obtained with larger cantilevers. This was modeled in Matlab with 3 points 
that represented the initial, central and final part of a given cantilever. A certain vertical noise $\left(z_{\text {noise }}\right)$ was applied to the $\mathrm{z}$ coordinate of each point and curvature was calculated based on the new position of the 3 points. The difference between the real and measured curvature is the measurement error. By repeating this simulation many times for each cantilever, and for many different curvatures, we obtained the measurement error as a function of cantilever length and curvature. Each level of noise yields a different plot. However, the simulated errors followed a similar trend which allowed us to derive approximate semi-empirical equations 14 and 15 that relate the cantilever length with the error in curvature estimation. We found that a vertical noise of $\pm 10 \mathrm{~nm}$ matched well the observed errors, and, in fact, it matched roughly the variability measured in the cantilever profiles.

$$
\text { Absolute curvature error }\left(\mathrm{mm}^{-1}\right) \approx \frac{\lambda}{L^{2}}
$$

where $\lambda$ is a coefficient determined experimentally that accounts for all the measurement error sources. In our case, $\lambda \approx 8 \times 10^{-11}$ matched well the worst-case observations.

$$
\text { Relative curvature error }(\%) \approx 100 \times \frac{\lambda}{K L^{2}}
$$

Remarkably, for a given $\lambda$ the absolute error (equation 14) does not depend on the curvature value being measured, but only on the length of the test cantilever, yielding a very simple formula, applicable for all cantilever curvature measurement techniques in general. The intuitive explanation for this simple relationship is that the shape of the cantilever follows a circumference, and the $\mathrm{z}$ displacement depends quadratically with the distance to the anchor (see equation 7). Therefore, an error in the measurement of the $\mathrm{z}$ position of cantilever tip will be less important the further it is from the anchor. The predicted curvature error derived from equation 14 is shown in table III for each cantilever length used in this work, assuming initial slopes equal to 0.1 and being the measured length $5 \mu \mathrm{m}$ smaller than the total length of the cantilever. Given that more accurate measurements can be achieved with longer cantilevers, these were the preferred option for the characterization tests presented in section $\mathrm{V}$, whenever the other limitations would allow it.

Note that the measured curvature variability $\left(\sigma_{K}\right)$ that appears on all tables and plots is composed of two components, namely, the real variability $\left(\sigma_{K}^{\text {real }}\right)$ plus the measurement error $\left(\sigma_{K}^{m e a s}\right)$, which is larger for shorter cantilevers (see table III). The two components are uncorrelated sources of dispersion so they satisfy, according to the Bienaymé formula:

$$
\sigma_{K}^{2}=\left(\sigma_{K}^{r e a l}\right)^{2}+\left(\sigma_{K}^{m e a s}\right)^{2}
$$

\section{RESULTS}

\section{A. Introduction}

The curvature of the released test cantilevers from both CMOS processes was analyzed. The measured parameter was curvature rather than radius of curvature given that, contrary to curvature values, which tend to be normally distributed (see
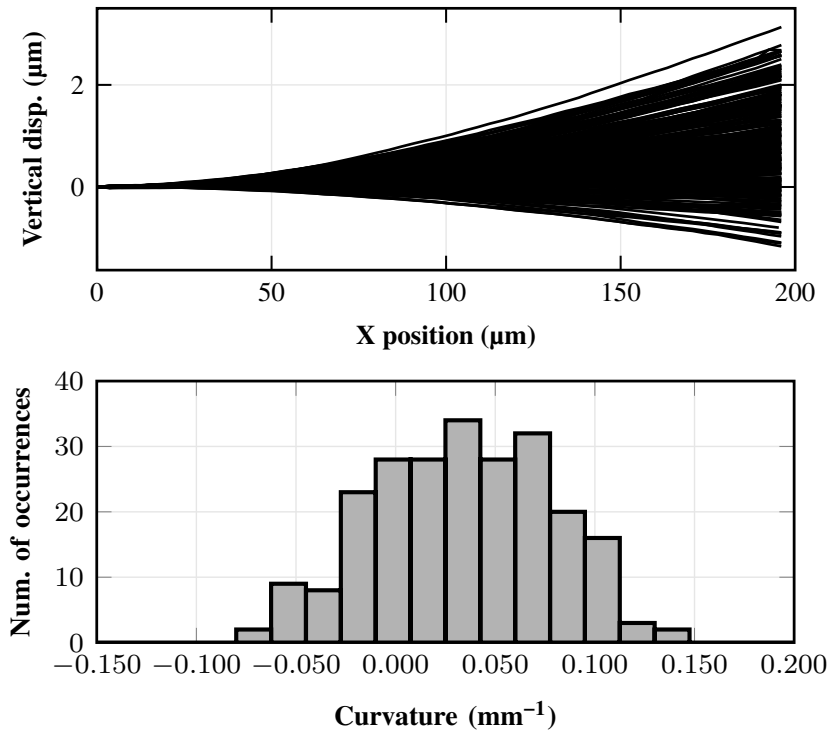

Figure 7: (Top) Profiles of all the 238 M345 $200 \mu \mathrm{m}$ cantilevers from Wafer 1, Lot 2, Run 3, LFoundry. (Bottom) Curvature distribution. Results from table $\mathrm{V}$ and the associated wafer map in figure $11 \mathrm{~b}$ show that the measured curvature was $0.034 \pm 0.044 \mathrm{~mm}^{-1}$. A gaussian distribution describes well the data. In this case, the average curvature is positive but there are cantilevers curved upwards and downwards in the same wafer. The slope at the anchoring point was $0.001 \pm 0.001$, close to zero.

bottom of figure 7) and are linearly dependent on the stress value (see equations 2 and 4), radius of curvature distributions do not provide an intuitive description of the physical system. For example, cantilevers from figure 7 have either large positive or negative curvature radius, which apparently yields non-representative variability results. On the other hand, all the associated curvature values are grouped around the mean.

Results are presented in tables IV and V for GF and LF, respectively. For each CMOS process, 8" wafers from different lots from the same run and from different runs were analyzed. The wide range of measurements performed can give the CMOS-MEMS designer an idea of the expected with-in-wafer (WIW), wafer-to-wafer (W2W), lot-to-lot (L2L), run-to-run $(\mathrm{R} 2 \mathrm{R})$ and CMOS process-to-process $(\mathrm{P} 2 \mathrm{P})$ variations.

For each set of measurements several parameters are specified, namely, the stack or metal layer characterized, mean curvature and variability across the sample, number and length of cantilevers measured, and other relevant information such as size of the sample (full wafer, quarter or small piece), run and lot number and other processing conditions. All wafers etched with Primaxx were baked after release except when indicated otherwise. Also, no backgrinding was applied except when indicated otherwise.

Note that the variability is the measured variability, which includes the measurement uncertainty (see equation 16). This uncertainty is usually not important compared to real variability except for the $20 \mu \mathrm{m}$-long cantilevers. It is important to note that the measured variability of small pieces or quarters of wafers is generally smaller than for full wafers, so care must be taken to extract conclusions from different sized samples. 


\begin{tabular}{|c|c|c|c|c|c|c|c|c|c|c|c|c|c|c|c|c|}
\hline & & M2 & M23 & M234 & M2345 & M23456 & M3 & M34 & M345 & M3456 & M4 & M45 & M456 & M5 & M56 & M6 \\
\hline WAFER 1 - no hot plate & Length $(\mu \mathrm{m})$ & & 20 & 80 & 100 & 150 & & 20 & 100 & 150 & & 20 & 150 & & 40 & \\
\hline LOT 2 & \#Cantilevers & & 52 & 52 & 44 & 34 & & 52 & 49 & 52 & & 52 & 52 & & 51 & \\
\hline GF - RUN 1 & Average $(1 / \mathrm{mm})$ & $\mathbf{K}$ & 2.997 & 0.613 & 0.333 & 0.181 & $\mathbf{K}$ & 1.339 & 0.267 & 0.013 & $\mathbf{K}$ & 1.919 & 0.212 & $\mathbf{K}$ & 0.880 & $\mathbf{K}$ \\
\hline MEMSSTAR $28 \mathrm{~min}$ & $\operatorname{Dev}(1 / \mathrm{mm})$ & & 1.039 & 0.277 & 0.207 & 0.101 & & 0.552 & 0.136 & 0.137 & & 0.719 & 0.201 & & 0.598 & \\
\hline WAFER 1 - after hot plate $30 \mathrm{~s}$ & Length $(\mu \mathrm{m})$ & & 20 & 80 & 100 & 150 & & 20 & 100 & 150 & & 20 & 150 & & 40 & \\
\hline $\begin{array}{l}\text { LOT } 2 \\
\text { L }\end{array}$ & \#Cantilevers & & 52 & 52 & 43 & 38 & & 51 & 49 & 51 & & 50 & 52 & & 52 & \\
\hline GF - RUN 1 & Average $(1 / \mathrm{mm})$ & $\mathbf{K}$ & 2.581 & 0.613 & 0.303 & 0.138 & $\mathbf{K}$ & 1.390 & 0.267 & 0.062 & $\mathbf{K}$ & 1.967 & 0.212 & $\mathbf{K}$ & 0.848 & $\mathbf{K}$ \\
\hline MEMSSTAR $28 \mathrm{~min}$ & Dev $(1 / \mathrm{mm})$ & & 1.017 & 0.277 & 0.173 & 0.109 & & 0.501 & 0.136 & 0.183 & & 0.623 & 0.201 & & 0.600 & \\
\hline WAFER 1 & Length $(\mu \mathrm{m})$ & 20 & 150 & 200 & 200 & 200 & 20 & 200 & 200 & 200 & 20 & 150 & 200 & 20 & 100 & 20 \\
\hline LOT 1 & \#Cantilevers & 40 & 50 & 48 & 49 & 50 & 49 & 50 & 50 & 50 & 49 & 50 & 51 & 50 & 50 & 51 \\
\hline GF - RUN 3 & Average $(1 / \mathrm{mm})$ & 7.864 & 0.999 & 0.313 & 0.096 & 0.132 & 6.049 & 0.575 & 0.129 & 0.182 & 6.625 & 0.595 & 0.392 & 8.050 & 1.277 & 8.093 \\
\hline PRIMAXX $36 \mathrm{~min}$ & Dev $(1 / \mathrm{mm})$ & 1.479 & 0.118 & 0.054 & 0.032 & 0.025 & 0.622 & 0.088 & 0.040 & 0.052 & 0.728 & 0.099 & 0.103 & 0.746 & 0.164 & 1.564 \\
\hline WAFER 2 & Length $(\mu \mathrm{m})$ & 20 & 150 & 200 & 200 & 200 & 20 & 150 & 200 & 200 & 20 & 150 & 200 & 20 & 150 & 20 \\
\hline LOT 1 & \#Cantilevers & 52 & 52 & 49 & 50 & 50 & 52 & 52 & 47 & 50 & 52 & 52 & 47 & 52 & 50 & 52 \\
\hline GF - RUN 3 & Average $(1 / \mathrm{mm})$ & 6.916 & 0.656 & 0.246 & 0.101 & 0.104 & 5.392 & 0.649 & 0.189 & 0.154 & 4.955 & 0.568 & 0.261 & 6.733 & 0.878 & 6.384 \\
\hline PRIMAXX $40 \mathrm{~min}$ & $\operatorname{Dev}(1 / \mathrm{mm})$ & 0.926 & 0.076 & 0.033 & 0.021 & 0.024 & 0.425 & 0.079 & 0.040 & 0.042 & 0.427 & 0.071 & 0.096 & 0.866 & 0.156 & 0.876 \\
\hline QUARTER OF WAFER 4 & Length $(\mu \mathrm{m})$ & 20 & 150 & 200 & 200 & 200 & 20 & 150 & 200 & 200 & 20 & 150 & 200 & 20 & 150 & 20 \\
\hline LOT 1 & \#Cantilevers & 12 & 11 & 11 & 12 & 11 & 12 & 12 & 12 & 10 & 12 & 12 & 12 & 12 & 11 & 12 \\
\hline GF - RUN 3 & Average $(1 / \mathrm{mm})$ & 8.291 & 1.005 & 0.289 & 0.145 & 0.138 & 7.272 & 0.656 & 0.227 & 0.209 & 7.621 & 0.731 & 0.330 & 8.779 & 1.107 & 7.329 \\
\hline PRIMAXX $55 \mathrm{~min}$ & $\operatorname{Dev}(1 / \mathrm{mm})$ & 0.337 & 0.092 & 0.046 & 0.028 & 0.025 & 0.591 & 0.082 & 0.051 & 0.075 & 0.766 & 0.049 & 0.129 & 0.966 & 0.170 & 1.415 \\
\hline QUARTER OF WAFER 4 & Length $(\mu \mathrm{m})$ & 20 & 150 & 200 & 200 & 200 & 20 & 150 & 200 & 200 & 20 & 150 & 200 & 20 & 150 & 20 \\
\hline LOT 1 & \#Cantilevers & 5 & 4 & 4 & 5 & 5 & 5 & 4 & 4 & 5 & 4 & 4 & 4 & 4 & 4 & 5 \\
\hline GF - RUN 3 & Average $(1 / \mathrm{mm})$ & 7.816 & 0.745 & 0.227 & 0.109 & 0.117 & 4.612 & 0.468 & 0.160 & 0.177 & 3.965 & 0.526 & 0.223 & 6.306 & 0.859 & 5.735 \\
\hline PRIMAXX $36 \mathrm{~min}$ & Dev $(1 / \mathrm{mm})$ & 0.262 & 0.041 & 0.027 & 0.013 & 0.018 & 0.114 & 0.058 & 0.015 & 0.033 & 0.260 & 0.040 & 0.053 & 0.198 & 0.096 & 0.972 \\
\hline
\end{tabular}

$\mathrm{K}=$ The profile of cantilevers could not be measured due to excessive initial slope at the anchor. The first run from GF (RUN 1) was experimental and several factors may have affected the measured curvature, so those particular results must be taken with caution.

Table IV: Curvature Measurement Results From Wafers From a CMOS 0.18 $\mu$ m Process From Global Foundries.

\section{B. Circularity}

The circularity of each of the $200 \mu \mathrm{m}$-long cantilever profiles shown in figure 7 was measured. The mean of the 238 circularity values obtained was $71 \mathrm{~nm}$ with a standard deviation of $\pm 35 \mathrm{~nm}$. Surface roughness and/or measurement noise accounted for $30-40 \%$ of the measured circularity given that circularity values around $20-30 \mathrm{~nm}$ were typically measured over flat metal surfaces. The cantilever profile deviations from a circular shape $(71 \mathrm{~nm})$ are small $(\sim 10 \%)$ compared to the tip displacement caused by the measured curvature $\left(0.034 \mathrm{~mm}^{-1}\right)$, which, according to equation 7 , is $680 \mathrm{~nm}$. This is a near worst case example. The same assessment was performed in all wafers, yielding circularity values between $1 \%$ and $10 \%$ (except in some cases where $\mathrm{K}$ was accidentally close to zero), showing that the cantilevers follow a very circular profile.

\section{Curvature of Stacks and Single Metals}

The Al-based BEOL metallization is typically sandwiched between two double thin layers of titanium (Ti) and titanium nitride (TiN). The larger coefficient of thermal expansion coefficient (CTE) of aluminum $\left(23.1 \times 10^{-6}{ }^{\circ} \mathrm{C}^{-1}\right)$, compared to those of $\mathrm{Ti}\left(8.6 \times 10^{-6}{ }^{\circ} \mathrm{C}^{-1}\right)$ and $\operatorname{TiN}\left(9.35 \times 10^{-6}{ }^{\circ} \mathrm{C}^{-1}\right)$, generally leads to tensile stresses for aluminum $(\mathrm{Al})$ and compressive stresses for $\mathrm{Ti}$ and TiN as a result of cooling down from higher processing temperature [25]. The final residual stresses create a bending moment that curls the cantilever upwards or downwards.

However, there are additional physical phenomena that affect cantilever curvature after release. For example, LFoundry single metals and their corresponding sub-layers have the same nominal thickness for all metal levels and corresponding sublayers, and no differences were measured in SEM images. However, the mean curvature of the metal levels is more negative the higher the metal level (see figure 8). This indicates that other factors apart from thickness are important in terms of curvature after release. These probably include residual stress variations dependent on temperature history of each metal level (see [26] and section V-K). As a matter of fact, lower metals like M2 go through more temperature cycles than higher ones like M5. This is a notable difference between LF and GF, as the curvature of GF single metals was independent of the metal level, large and positive.

Measured curvature of cantilevers from several LF and GF runs (one of them being a full CMOS-process that included the FEOL) is displayed in figure 8 . The mean curvature of single metals (a few $\mathrm{mm}^{-1}$ ) and their WIW variations (generally between 0.5 and $1.0 \mathrm{~mm}^{-1}$ ) were generally very large compared to other MEMS processes [27]. In addition, their W2W, L2L and $\mathrm{R} 2 \mathrm{R}$ variations were also in the same order of magnitude, around $1 \mathrm{~mm}^{-1}$, roughly. Unfortunately, this imposes serious restrictions to design competitive MEMS with single metals. As a result of single metals curvature (positive for GF and generally negative for LF), the vast majority of stacks from all the runs from GF showed an average positive curvature, while negative curvatures were more predominant in the case of LF, as the first right hand side (RHS) term of equation 8 predicts. For stacks of metal layers results consistently showed that not only the mean curvature but also, and more importantly, their WIW, L2L, R2R and P2P variations were significantly reduced with respect to single metal layers. For example, for the full process wafer from LF, both average curvature and WIW variations of M2345 $\left(-0.091 \pm 0.040 \mathrm{~mm}^{-1}\right)$ were reduced around 20 times with respect to M2 $\left(-1.903 \pm 0.766 \mathrm{~mm}^{-1}\right)$, and mean curvature was reduced around 64 times when compared to M5 $\left(-5.865 \pm 0.829 \mathrm{~mm}^{-1}\right)$. This improvement is greatly correlated with the number of stacked layers, which is explained by equation 8 , as the denominators are the increased bending stiffness of the stacks, presented in table II. Curvature reduction varied from case to case, achieving more than 2 orders of reduction in some cases (see M2345 and M5 from wafer 2, lot 1, run 4, LF) and around 1 order of magnitude in others (see M2345 and M3 from wafer 1, lot 3, run 3, LF). With-in-wafer variability reductions for 4- 


\begin{tabular}{|c|c|c|c|c|c|c|c|c|c|c|c|c|c|c|c|c|}
\hline & & M2 & M23 & M234 & M2345 & M23456 & M3 & M34 & M345 & M3456 & M4 & M45 & M456 & M5 & M56 & M6 \\
\hline HALF OF WAFER 2 & Length $(\mu \mathrm{m})$ & 20 & 60 & 200 & 200 & & 20 & 80 & 80 & 100 & 20 & 60 & & 20 & 60 & 60 \\
\hline LOT 1 & \#Cantilevers & 47 & 51 & 50 & 50 & & 51 & 50 & 51 & 52 & 52 & 52 & & 52 & 52 & 52 \\
\hline LF - RUN 1 & Average $(1 / \mathrm{mm})$ & 0.700 & 0.111 & 0.105 & 0.023 & NA & -0.516 & -0.159 & -0.257 & 1.223 & -1.687 & -0.877 & NA & -3.828 & 2.420 & 3.054 \\
\hline PRIMAXX & $\operatorname{Dev}(1 / \mathrm{mm})$ & 1.827 & 0.245 & 0.103 & 0.065 & & 2.148 & 0.258 & 0.107 & 0.164 & 1.964 & 0.235 & & 1.926 & 0.372 & 0.457 \\
\hline QUARTER OF WAFER 1 & Length $(\mu \mathrm{m})$ & & & & & & 20 & 100 & 80 & 200 & 20 & 80 & 200 & $20-40$ & 200 & 100 \\
\hline LOT 1 & \#Cantilevers & & & & & & 62 & 60 & 58 & 62 & 62 & 61 & 61 & 59 & 60 & 61 \\
\hline LF - RUN 2 & Average $(1 / \mathrm{mm})$ & NR & NR & NR & NR & NR & -1.281 & 0.008 & -0.183 & 0.376 & -2.397 & -0.629 & 0.512 & -5.934 & 0.725 & -0.175 \\
\hline PRIMAXX 25 min & Dev $(1 / \mathrm{mm})$ & & & & & & 1.094 & 0.050 & 0.090 & 0.094 & 0.660 & 0.148 & 0.104 & 1.551 & 0.110 & 0.082 \\
\hline QUARTER OF WAFER 2 & Length $(\mu \mathrm{m})$ & 20 & 40 & 100 & 60 & 150 & 20 & 80 & 40 & 150 & 20 & 60 & 150 & 20 & 200 & 100 \\
\hline LOT 1 & \#Cantilevers & 52 & 52 & 51 & 45 & 51 & 52 & 44 & 49 & 40 & 51 & 45 & 49 & 52 & 51 & 52 \\
\hline LF - RUN 2 & Average $(1 / \mathrm{mm})$ & -1.864 & -0.338 & -0.030 & -0.144 & 0.241 & -3.463 & -0.129 & -0.293 & 0.311 & -3.933 & -0.886 & 0.430 & -7.860 & 0.678 & -0.335 \\
\hline PRIMAXX $35 \mathrm{~min}$ & Dev $(1 / \mathrm{mm})$ & 0.418 & 0.085 & 0.050 & 0.055 & 0.057 & 0.581 & 0.097 & 0.138 & 0.101 & 0.685 & 0.180 & 0.136 & 0.910 & 0.113 & 0.135 \\
\hline QUARTER OF WAFER 1 & Length $(\mu \mathrm{m})$ & & & 200 & 200 & 150 & 20 & 200 & 200 & 150 & 20 & 100 & 100 & 20 & 100 & 60 \\
\hline LOT 1 & \#Cantilevers & & & 87 & 85 & 89 & 87 & 81 & 88 & 88 & 84 & 81 & 87 & 87 & 89 & 88 \\
\hline LF - RUN 3 & Average $(1 / \mathrm{mm})$ & NR & NR & 0.079 & 0.167 & 0.673 & -1.581 & 0.105 & 0.184 & 0.849 & -3.366 & -0.186 & 1.040 & -4.325 & 1.400 & 2.986 \\
\hline PRIMAXX $25 \mathrm{~min}$ & Dev $(1 / \mathrm{mm})$ & & & 0.061 & 0.040 & 0.075 & 0.573 & 0.079 & 0.038 & 0.079 & 0.701 & 0.100 & 0.165 & 0.736 & 0.270 & 0.353 \\
\hline QUARTER OF WAFER 2 & Length $(\mu \mathrm{m})$ & 20 & 60 & 200 & 200 & 200 & 20 & 80 & 150 & 200 & 40 & 80 & 150 & 40 & 100 & 60 \\
\hline LOT 1 & \#Cantilevers & 83 & 86 & 85 & 85 & 84 & 86 & 83 & 86 & 85 & 86 & 86 & 86 & 85 & 86 & 86 \\
\hline LF - RUN 3 & Average $(1 / \mathrm{mm})$ & -1.765 & 0.012 & 0.033 & 0.072 & 0.554 & -2.758 & -0.229 & -0.046 & 0.689 & -2.733 & -0.410 & 0.898 & -3.803 & 1.329 & 2.885 \\
\hline PRIMAXX 35 min & $\operatorname{Dev}(1 / \mathrm{mm})$ & 0.540 & 0.065 & 0.025 & 0.015 & 0.060 & 0.532 & 0.078 & 0.031 & 0.081 & 0.180 & 0.078 & 0.155 & 0.282 & 0.222 & 0.266 \\
\hline WAFER 1 & Length $(\mu \mathrm{m})$ & 20 & 100 & 200 & 200 & 200 & 20 & 100 & 200 & 200 & 20 & 100 & 150 & 20 & 150 & 200 \\
\hline LOT 2 & \#Cantilevers & 235 & 237 & 232 & 236 & 239 & 234 & 238 & 233 & 238 & 235 & 232 & 238 & 236 & 238 & 238 \\
\hline LF - RUN 3 & Average $(1 / \mathrm{mm})$ & 0.477 & 0.289 & 0.106 & 0.111 & 0.416 & -0.885 & -0.002 & 0.034 & 0.472 & -2.612 & -0.176 & 0.566 & -4.288 & 0.555 & 0.235 \\
\hline PRIMAXX $30 \mathrm{~min}$ & $\operatorname{Dev}(1 / \mathrm{mm})$ & 0.749 & 0.135 & 0.054 & 0.031 & 0.077 & 0.685 & 0.159 & 0.044 & 0.088 & 0.938 & 0.145 & 0.140 & 0.869 & 0.213 & 0.071 \\
\hline WAFER 1 & Length $(\mu \mathrm{m})$ & 80 & 40 & 40 & 60 & 150 & 20 & 60 & 60 & 150 & 20 & 60 & 100 & 20 & 80 & 60 \\
\hline LOT 3 & \#Cantilevers & 113 & 114 & 122 & 115 & 119 & 119 & 119 & 119 & 119 & 119 & 115 & 119 & 119 & 119 & 119 \\
\hline LF - RUN 3 & Average $(1 / \mathrm{mm})$ & 1.913 & -0.280 & -0.284 & -0.225 & 0.707 & -1.631 & -0.402 & -0.285 & 0.955 & -2.404 & -0.968 & 1.306 & -4.678 & 2.083 & 2.891 \\
\hline PRIMAXX $32 \mathrm{~min}$ & $\operatorname{Dev}(1 / \mathrm{mm})$ & 0.858 & 0.218 & 0.145 & 0.081 & 0.121 & 0.928 & 0.205 & 0.110 & 0.160 & 1.071 & 0.199 & 0.222 & 1.177 & 0.355 & 0.322 \\
\hline WAFER 2 & Length $(\mu \mathrm{m})$ & 20 & 40 & 80 & 100 & 150 & 20 & 60 & 100 & 150 & 20 & 60 & 100 & 20 & 80 & 60 \\
\hline LOT 1 & \#Cantilevers & 241 & 240 & 241 & 241 & 226 & 240 & 241 & 241 & 230 & 241 & 240 & 232 & 240 & 232 & 232 \\
\hline LF - RUN 4 & Average $(1 / \mathrm{mm})$ & -0.134 & -0.120 & -0.008 & -0.040 & 0.608 & -2.461 & -0.133 & -0.141 & 0.829 & -3.663 & -0.738 & 1.188 & -5.080 & 1.820 & 3.226 \\
\hline PRIMAXX $32 \mathrm{~min}$ & $\operatorname{Dev}(1 / \mathrm{mm})$ & 0.873 & 0.132 & 0.045 & 0.028 & 0.076 & 0.860 & 0.099 & 0.049 & 0.102 & 0.995 & 0.121 & 0.177 & 1.099 & 0.299 & 0.314 \\
\hline WAFER 3 (Backgrinded to $500 \mu \mathrm{m}$ ) & Length $(\mu \mathrm{m})$ & 40 & 60 & 80 & 80 & 100 & 20 & 100 & 100 & 100 & 20 & 100 & 100 & 20 & 100 & 60 \\
\hline LOT 1 & \#Cantilevers & 233 & 233 & 227 & 228 & 220 & 206 & 232 & 227 & 232 & 239 & 230 & 226 & 233 & 229 & 231 \\
\hline LF - RUN 4 & Average $(1 / \mathrm{mm})$ & 0.490 & -0.084 & -0.044 & 0.042 & 0.632 & -1.441 & -0.174 & 0.021 & 0.866 & -3.048 & -0.370 & 1.202 & -4.147 & 1.763 & 2.874 \\
\hline PRIMAXX $32 \mathrm{~min}$ & $\operatorname{Dev}(1 / \mathrm{mm})$ & 0.687 & 0.115 & 0.047 & 0.039 & 0.085 & 0.842 & 0.069 & 0.057 & 0.123 & 1.068 & 0.117 & 0.179 & 1.102 & 0.256 & 0.363 \\
\hline WAFER 1 (Backgrinded to $500 \mu \mathrm{m}$ ) & Length $(\mu \mathrm{m})$ & 20 & 20 & 40 & 80 & 200 & 20 & 40 & 80 & 200 & 20 & 60 & 150 & 20 & 200 & 100 \\
\hline LOT 1 & \#Cantilevers & 243 & 238 & 240 & 236 & 242 & 243 & 244 & 243 & 244 & 244 & 244 & 244 & 244 & 242 & 243 \\
\hline LF - RUN 5 (FULL PROCESS) & Average $(1 / \mathrm{mm})$ & -1.903 & -0.569 & -0.296 & -0.091 & 0.428 & -3.030 & -0.771 & -0.235 & 0.480 & -4.536 & -0.868 & 0.521 & -5.865 & 0.437 & -0.384 \\
\hline PRIMAXX $30 \mathrm{~min}$ & $\operatorname{Dev}(1 / \mathrm{mm})$ & 0.766 & 0.218 & 0.160 & 0.040 & 0.104 & 0.645 & 0.310 & 0.083 & 0.130 & 1.242 & 0.136 & 0.173 & 0.829 & 0.114 & 0.089 \\
\hline
\end{tabular}

$\mathrm{NR}=$ Not released. $\mathrm{NA}=$ Not available.

Table V: Curvature Measurement Results from WaFers From a CMOS $0.18 \mu$ m Process From L-Foundry.

metals with respect to single metals typically ranged from 20 to 40 times. Measured stack curvatures cannot be accurately predicted exclusively by the first RHS term of equation 8, which only takes into account the curvature of single metals and the increased bending stiffness. This indicates that the average stress mismatch between layers plays an important role in the observed curvatures, according to equation 8 , if the used $\mathrm{W}$ vias do not affect stack curvature.

Theoretically, when the $\mathrm{W}$ vias effect is negligible $\left(M_{W i} \rightarrow\right.$ 0 ), as for the standard $\mathrm{W}$ vias arrays used in this work, the average stress mismatch between single metal layers may be calculated by solving for the stress $\left(\sigma_{i}\right)$ in equation 8. For example, the stress mismatch between M3 and M2 $\left(\sigma_{3}-\sigma_{2}\right)$ can be derived from the M2, M3 and M23 curvatures:

$$
\sigma_{3}-\sigma_{2}=\left(K_{\text {stack }}^{23} \frac{E I_{\text {stack }}}{E I}-K_{2}-K_{3}\right) \frac{E t^{2}}{6(t+g)}
$$

Once the stress mismatch value is known, the associated strain mismatch value, which does not depend on the assumed Young's Modulus $(E)$, can be calculated by dividing by $E$. Results yielded strain mismatch values between adjacent layers lower than 700 ppm for both GF and LF processes. However, under sufficiently high stress/strain mismatch, the applicability of linear equations 8 and 17 is limited by non-linear effects such us localized plastic deformation or partial delamination near the anchor of the $\mathrm{W}$ vias. Plastic deformation is more plausible given that no delamination has been observed in SEM images. Either of these mechanisms allow greater expansion/contraction mismatch between the layers, which is absorbed by shear deformation of the stack, relieving part of the stress mismatch that, otherwise, would have led to larger curvature. As a consequence, equation 17 is expected to underestimate stress/strain mismatch. A similar equation can be derived for 3-metal stacks. In that case, numerical simulations show that the Von Mises stress levels near the $\mathrm{W}$ via anchor are even larger when more layers are stacked. This is reasonable given that, when more layers are stacked, the curvature is smaller, so the stress mismatch is relieved to a lesser extent. In fact, curvature values from tables IV and $\mathrm{V}$ confirm that 3-metal cantilevers curve less than expected if stress/strain mismatches derived from 2-metal cantilevers are assumed, which can be explained by either of the two aforementioned non-linear mechanisms.

\section{Variability}

For each metal there was 1 test structure per reticle, so within-reticle variations are not included in this work. Curvature varies smoothly across the wafers as can be observed in the wafer maps of figures 10 and 11 for LF process. Matching of curl of adjacent test cantilevers of different lengths from the 


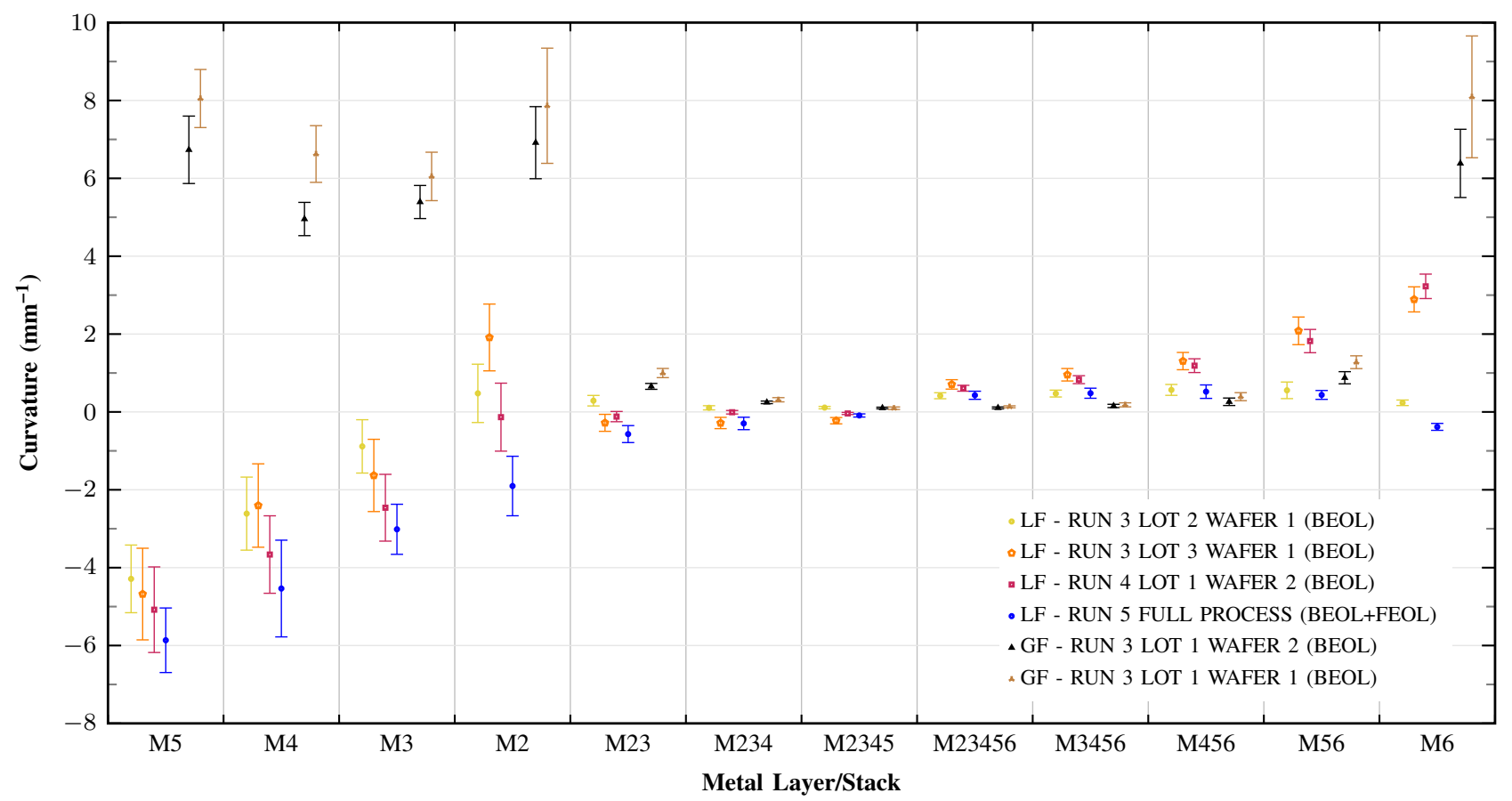

Figure 8: Average curvature and variability of some metal stacks used in 6 different wafers. Stacking of identical metal layers led to a reduced variability and average curvature, which is the typical behavior observed in all the runs analyzed in this work. Stacking of dissimilar metal layers (M6 and others) may not be as efficient (compare M2345 with M23456).

same test array was good (see figures $2 \mathrm{~b}$ and 6 ), generally not much larger than the measurement error of table III. This is in agreement with the results reported in [9].

The thickness of the Al-based central layer and the bottom and top thin Ti/TiN layers was measured with SEM. The measured thicknesses are in table I. Thickness variations of the $\mathrm{Ti}$ and/or TiN layers lead to curvature variations after cool down from the deposition or other processes temperature due to CTE mismatch with aluminum. Unfortunately, accurate assessments are not possible given that, the relative proportion of Ti and TiN could not be determined and complex phenomena, like plastic deformations $[26,28]$ and creep (see section $\mathrm{V}-\mathrm{K}$ ), or material properties dependence with thickness [29] may be taking place. However, in order to approximately understand the importance of Ti/TiN thickness variations on single metal curvature due to CTE mismatch after cool down, the following 2 cases were considered: one in which the Ti layer $(\mathrm{E}=116 \mathrm{GPa})$ is $15 \mathrm{~nm}$ thick, and the rest of the Ti/TiN layer is pure $\mathrm{TiN}(\mathrm{E}=476 \mathrm{GPa}[30])$; and another identical to the previous one but with a $10 \%$ thickness reduction of the top TiN layer. Then, finite-element-analysis (FEA) was employed to assess the effect of a $250{ }^{\circ} \mathrm{C}$ cool down, which is not uncommon in CMOS BEOL process steps, which can even go up to $450^{\circ} \mathrm{C}$ [31]. FEA yielded a curvature difference between the 2 cases of $0.37 \mathrm{~mm}^{-1}$ for LF single metals, and of $0.27 \mathrm{~mm}^{-1}$ for GF single metals. The obtained results are approximately linear with thickness variations and temperature cool down: for example, $1 \%$ thickness variations cause a similar mismatch effect to $25^{\circ} \mathrm{C}$ variations. These results suggest that thickness variations of the Ti/TiN layers can be playing an important role in the observed single metals curvature variability, which is around $1 \mathrm{~mm}^{-1}$ in order of magnitude.

Contrary to single metals, stack curvature also depends on the gap between metals as described by equation 8 , where the gap variability changes the value of the bending stiffness $\left(I E_{\text {stack }}\right)$. This effect is important as, for example, equation 8 predicts a 2 -metal stack curvature variability of $13 \%$ for a gap variability of $10 \%$ and a metal thickness variability of $5 \%$, which is not a bad scenario in conventional metal interconnect. Note that this is just taking into account bending stiffness variations. If single metal curvature variability and measurement errors are included, measured variability exceeds the predicted value in many cases. This probably indicates that stress mismatch variability and plastic deformations should also be considered. On the other hand, no evidence of curvature variability due to the vHF etch was found given that etch time differences as large as 21 minutes did not affect curvature noticeably (see section V-F) and vHF etch WIW variations are around $\pm 1 \mathrm{~min}$ [17]. So, while thickness and temperature history can be important for the mean curvature and variability of single metals, gap uniformity and stress mismatch are also important for the curvature of stacks.

Curvature patterns of single metals tend to be transfered to the stacks in LF wafers (see figures 10 and 11), highlighting the importance of the first RHS term of equation 8 . Singlemetal curvature typically follows a radial-like distribution. Despite stacks of metals also show radial distributions frequently, others like annular and cross-wafer are also observed, generally superimposed to a radial-like background distribution.

Chemical-mechanical-polishing (CMP) is a significant source of IMD oxide thickness/gap variability. It can induce 


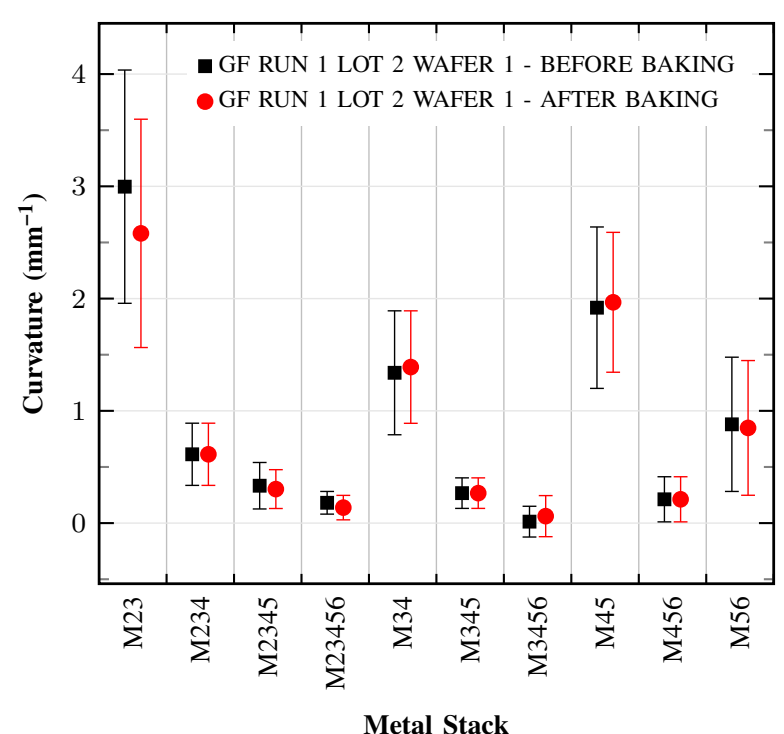

Figure 9: Comparison of the curvature before and after a baking step that consisted on $200^{\circ} \mathrm{C}$ during 30 seconds.

complex patterns of radial thickness variation as the observed annular patterns for curvature of stacks. Many MEMS comprise large metal structures that need to break maximum metal size rules of standard CMOS design. This can accentuate the CMP adverse effects as high density metal areas may be overpolished and end up thinner, so dummy metal filling is used to reduce thickness dependence on layout pattern. Also, temperature gradients in radial or cross-wafer directions may be present in process chambers, and spinning of wafers in lithography, and wafer cleaning steps may introduce radial variations [32]. In any case, determination of the exact source of curvature variability would require specifically designed experiments.

\section{E. Baking Step Effects}

An important question is if the baking step of the CMOSMEMS process modifies the curvature of the BEOL metal layers. For this purpose, the curvature characterization was performed on a randomly selected wafer before and after the baking step, which in this case consisted on putting the wafer on a hot plate at approximately $200^{\circ} \mathrm{C}-250^{\circ} \mathrm{C}$ for 30 seconds, which was enough to remove the residues. Characterization results (see figure 9) showed that the curvature did not significantly change. As we shall see, this will be in accordance to the results of section V-K regarding temperature effects on curvature. Some variation is observed for M23 composites, but this is attributed to the reduced accuracy of the $20 \mu \mathrm{m}$ long cantilevers used for the characterization in this case.

\section{F. Curvature versus Time}

Limited measurements were performed in this respect, but significant curvature changes were not observed with time. Also, in the case of GF wafer 1 from lot 2, run 1, the full wafer was measured before and after the baking step: 4.5 weeks passed between measurements. They were stored at
$45 \%$ relative humidity. No significant changes were observed. Silicon oxide stress can change significantly due to moisture absorption [33, 34], potentially leading to curvature variations of oxide structures with time. However, the cantilevers of this work do not leave any oxide exposed to ambient air.

\section{G. BEOL versus $F E O L+B E O L$}

Processing wafers with only the BEOL is significantly cheaper than processing full-CMOS wafers, so determining if BEOL wafers are sufficient to replicate the behavior of full-CMOS wafers is very important. For this purpose, both full-CMOS and BEOL wafers were measured. In figure 8 and table $\mathrm{V}$ no noticeable differences can be observed between the full-CMOS run and BEOL runs. This result agrees with the fact that the BEOL is processed after the FEOL, so the latter should not affect the former.

Only M6 curvature changed from a typically large positive curvature in BEOL runs to a small negative one, as happened for BEOL run 2 and lot2 from run 3, of LFoundry (see table IV and figure 8). In both cases, some passivation oxide over M6 was remaining after the pad opening step, leaving the Ti/TiN layer and aluminum of M6 intact, contrary to what happened in those cases that showed large M6 curvature. The thickness of the Ti/TiN and Al layers of M6 was measured with FIB cuts, resulting in $40 \pm 15 \mathrm{~nm}$ and $820 \pm 15 \mathrm{~nm}$, respectively. Numerical simulations showed that a composite beam made of a $40 \mathrm{~nm}$ layer of TiN below a $820 \mathrm{~nm}$ thick layer of $\mathrm{Al}$, and no TiN on top of the Al, curves upwards $3.0 \mathrm{~mm}^{-1}$ when cooled down $200^{\circ} \mathrm{C}$ from an initially free-stress state, simulating grossly the cool down after a generic deposition process. If the TiN layer were substituted by pure $\mathrm{Ti}$, identical upwards curling would be achieved with a $430{ }^{\circ} \mathrm{C}$ cool down. Other combinations of both materials yield similar numbers. Therefore, Ti/TiN etch, and probably some $\mathrm{Al}$ etch, are thought to be the source of the M6 curvature differences found.

\section{H. Release Process and Etch Time}

The studied wafers were etched with machines from two different vendors, namely, MEMSstar and Primaxx. Gathered data is insufficient to draw an accurate conclusion regarding the curvature dependence on the etching machine and method, but it can be affirmed that no dramatic differences were observed.

Although no specific experiment was set to assess the effect of etching time on curvature, some conclusions can be extracted from several quarters of the same wafers that were etched with the Primaxx machine for different times like the two quarters of wafer 4, lot 1, run 3 from GF: one quarter was etched for $36 \mathrm{~min}$ and the other one for $55 \mathrm{~min}$ (see table IV). Observed curvature differences between both samples fall within the normal variation between the quarters of a wafer. Also, the bottom surface of the cantilevers from figure 4 were exposed to vHF for different times, in average, depending on the cantilever width. This difference can be estimated by using the vHF etch rate $\left(0.25 \mu \mathrm{m} \mathrm{min}^{-1}\right)$ reported in [17]. In average, the bottom surface of the $30 \mu \mathrm{m}$-wide cantilever was exposed 


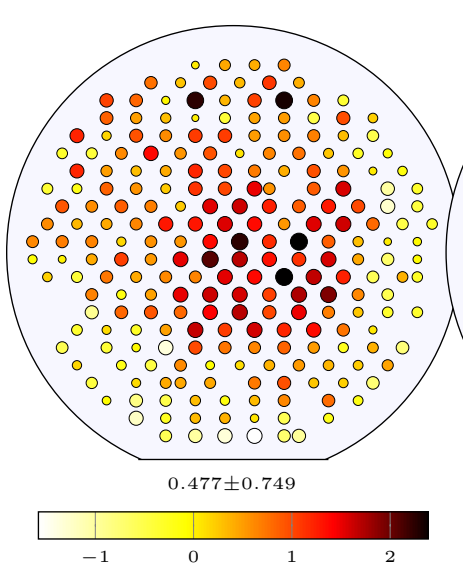

(a) M2

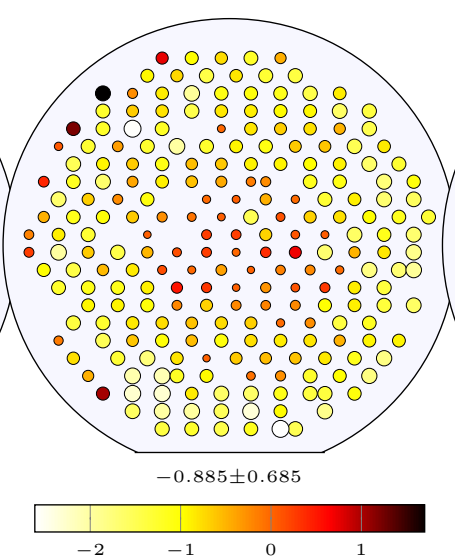

(b) M3

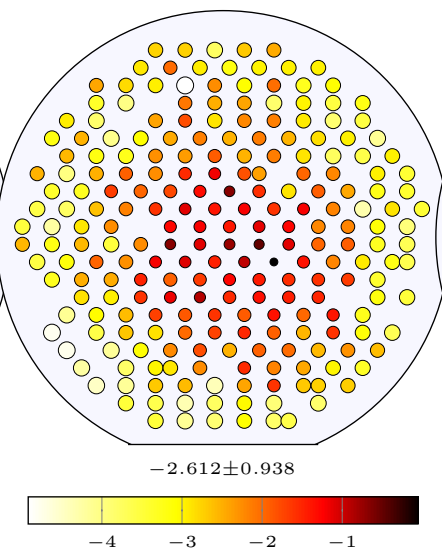

(c) M4

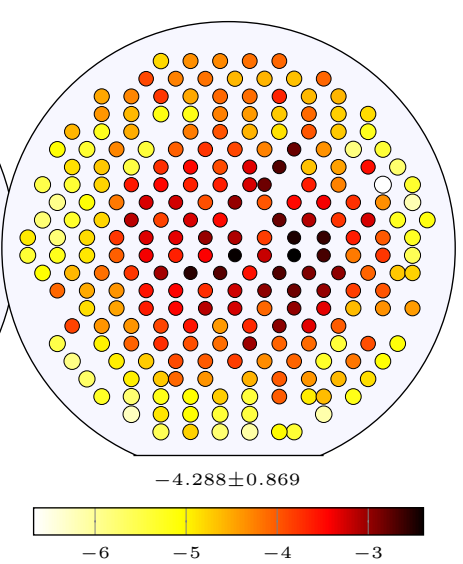

(d) M5

Figure 10: Curvature distribution for some single metals: Wafer 1, Lot 2, Run 3 from LFoundry. Area of markers is proportional to the absolute curvature and was normalized with the maximum absolute curvature value over each wafer.

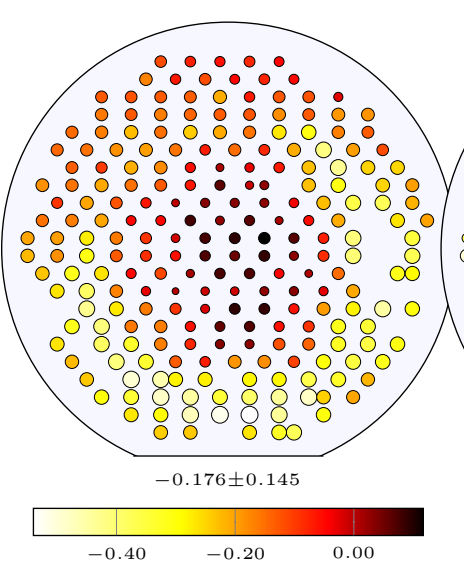

(a) M45

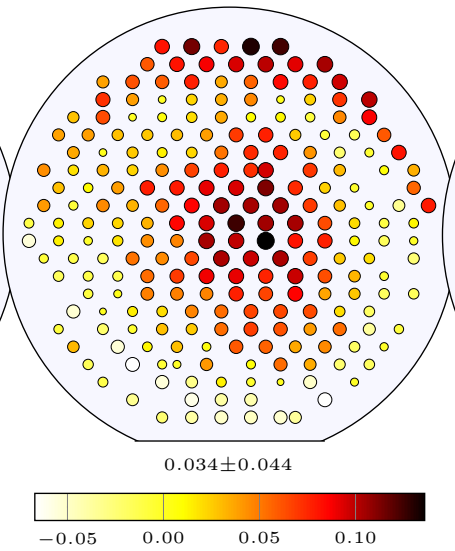

(b) M345

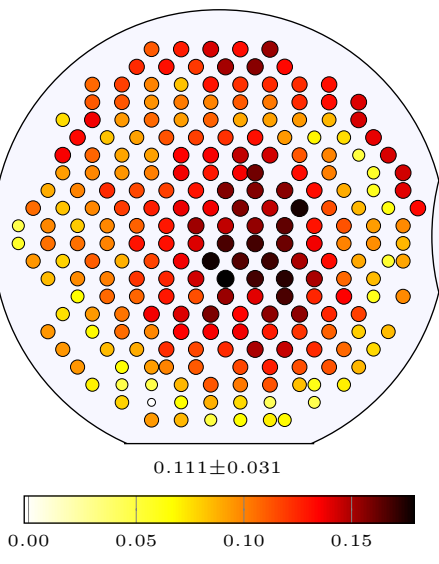

(c) M2345

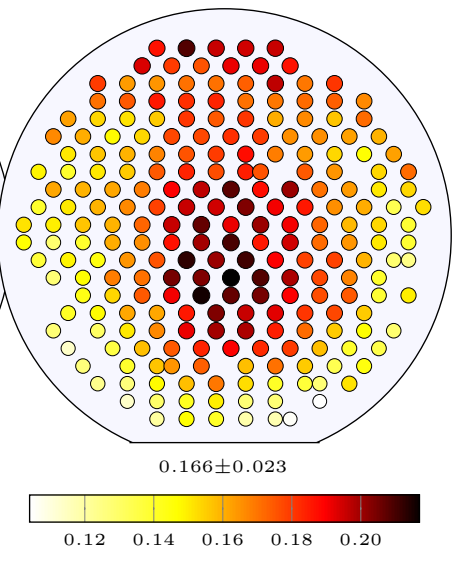

(d) M2345-O

Figure 11: Curvature distribution for some stacks of metals: Wafer 1, Lot 2, Run 3 from LFoundry. Area of markers is proportional to the absolute curvature and was normalized with the value of the maximum absolute curvature over each wafer. Note the scale is different to highlight the differences in wafers with lower variations.

to vHF 25 min longer than the $5 \mu \mathrm{m}$-wide cantilever. However, no significant curvature differences are observed in the picture.

Although curvature dependence on etching time cannot be assessed accurately with the extant data, it is by all means significantly less important than other sources of variability. This is in agreement with the vHF high selectivity of silicon oxide to the metals that form the test cantilevers [17, 35-37].

\section{Backgrinding}

Finally, another important step in wafer processing is the backgrinding, which is useful for reducing the thickness of the wafer prior to subsequently packaging steps. Wafer 3 from lot 1 , run 4 from LF was backgrinded to a thickness of $500 \mu \mathrm{m}$ (see table V) and no noticeable effects were observed when compared to other wafers from the same lot than were not backgrinded.

\section{J. Effect of W Via Desing on Curvature}

In order to assess the effect of the $\mathrm{W}$ via design on the curvature of stacks of metal, test cantilevers with 4 different via designs were fabricated in M34, M234, M345 and M2345 in two runs. The 4 designs tested and the results obtained are shown in table VI. Via design 1 is the one used in the test cantilevers presented in this work: a standard rectangular array with a via width and via spacing of approximately $0.3 \mu \mathrm{m}$. Via design 2 is just a long continuous via that runs along the perimeter of the cantilever, enclosing the IMD oxide between the metal layers. Via design 3 is an array of intersecting perpendicular long continuous vias, which also encloses some IMD oxide between the metal layers. Via design 4 is like design 2, but also with the standard rectangular array of vias.

Results showed a strong correlation between mean curvature and via design. Numerical simulations of the $W$ via design 1 showed that the $\mathrm{W}$ vias residual stress does not change the curvature of the stack (third RHS term of equation 8 is zero). The non continuity of the individual vias along the length of the beam is the reason for this effect. This is in agreement with their negligible contribution to $E I_{\text {stack }}(\alpha=0)$ as mentioned in section IV-B, and with the measurements shown at the bottom half of table VI, which yielded very similar curvatures for designs 2 and 4 (note that for the M34 stack 


\begin{tabular}{|c|c|c|c|c|c|c|c|c|c|c|}
\hline & & $\begin{array}{c}\text { M234 } \\
::::: \\
: 10::\end{array}$ & $\begin{array}{l}\text { M234 } \\
\text { (2) }\end{array}$ & 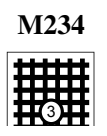 & 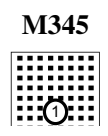 & 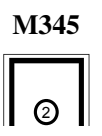 & $\begin{array}{c}\text { M345 } \\
\text { \#\# } \\
\text { \#3\# }\end{array}$ & $\begin{array}{l}\text { M2345 } \\
:::: a \\
: 11::\end{array}$ & $\begin{array}{l}\text { M2345 } \\
\text { (2) }\end{array}$ & $\begin{array}{l}\text { M2345 } \\
\text { \#册 } \\
\text { 3\# }\end{array}$ \\
\hline WAFER 1 & Length $(\mu \mathrm{m})$ & 200 & 200 & 150 & 200 & 200 & 150 & 200 & 200 & 150 \\
\hline LOT 2 & \#Cantilevers & 232 & 238 & 208 & 233 & 238 & 219 & 236 & 238 & 166 \\
\hline LF - RUN 3 & Ave $\left(\mathrm{mm}^{-1}\right)$ & 0.106 & 0.262 & 1.020 & 0.034 & 0.232 & 0.887 & 0.111 & 0.166 & 0.644 \\
\hline \multirow[t]{2}{*}{ PRIMAXX $30 \mathrm{~min}$} & $\operatorname{Dev}\left(\mathrm{mm}^{-1}\right)$ & 0.054 & 0.061 & 0.092 & 0.044 & 0.058 & 0.088 & 0.031 & 0.023 & 0.112 \\
\hline & & 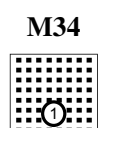 & \begin{tabular}{|r|} 
M34 \\
(2)
\end{tabular} & 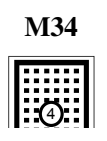 & 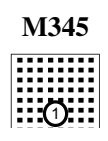 & $\begin{array}{l}\text { M345 } \\
\begin{array}{|r|}\mid \text { (2) }\end{array}\end{array}$ & 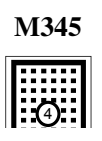 & $\begin{array}{l}\text { M3456 } \\
:::: a: \mid \\
: \vdots 1):\end{array}$ & $\begin{array}{l}\text { M3456 } \\
\text { (2) }\end{array}$ & 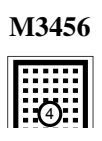 \\
\hline HALF OF WAFER 2 & Length $(\mu \mathrm{m})$ & 80 & 80 & 60 & 80 & 150 & 100 & 100 & 150 & 100 \\
\hline LOT 1 & \#Cantilevers & 50 & 51 & 52 & 51 & 48 & 49 & 52 & 52 & 51 \\
\hline LF - RUN 1 & Ave $\left(\mathrm{mm}^{-1}\right)$ & -0.159 & 0.128 & -0.005 & -0.257 & 0.119 & 0.119 & 1.223 & 0.804 & 0.879 \\
\hline PRIMAXX 35 min & $\operatorname{Dev}\left(\mathrm{mm}^{-1}\right)$ & 0.258 & 0.316 & 0.325 & 0.107 & 0.120 & 0.113 & 0.164 & 0.063 & 0.073 \\
\hline
\end{tabular}

Table VI: Comparison Table. Effect of Tungsten (W) Vias on Curvature of the Metal Stack.

the variability is large, so assessment is not so accurate as with the 3-metal stacks). Although in design 4 the $\mathrm{W}$ vias are joined with IMD oxide, which provides some continuity between the vias, the IMD oxide effect is small, as revealed by the similar bending stiffness (within 10\%) and $\alpha$ value of designs 2 and 4. Designs 2, 3 and 4, which run continuously along the cantilever length, do modify the stack curvature. In fact, FEA showed that the curvature change due to residual stress gradients of the $\mathrm{W}$ vias (including their corresponding Ti/TiN layers), or due to mismatch between different levels of $\mathrm{W}$ vias, is 2.5-3.0 times more pronounced for design 3 than for designs 2 and 4 . Measurements confirmed this qualitatively, but showed a greater curvature difference $(>4)$ between designs 3 and 2. Given that no significant thickness variations of the Ti/TiN layers of the single metal layers were observed in SEM images, the discrepancy could be related to localized plastic deformations or partial delaminations near the anchor of the $\mathrm{W}$ vias, as curvature data suggested when the residual stress mismatch between single layers was evaluated in section V-C, but it is not possible to confirm this with the extant data.

From a design point of view, the most important conclusion is that the curvature of the metal stacks may be tailored up to some degree by the appropriate choice of the $\mathrm{W}$ via design used to connect the metal layers. This comes with associated design complexity as the formulas for displacement and resonant frequency of solid cantilevers are no longer applicable. However, in section IV-B we show analytical equations to calculate the bending stiffness of stacked cantilevers with arbitrary $\mathrm{W}$ vias design. The coefficient $\alpha$ should be empirically or numerically determined, and it is approximately $0.00,0.22,0.44$ and 0.25 for $\mathrm{W}$ via designs $1,2,3$ and 4 , respectively, according to FEA. The values are the same for LF and GF processes given that the via size and spacings are similar. Once the bending stiffness is known, the resonant frequency or spring constant can be readily calculated as described in $[38,39]$. Also, FEA can be simplified using equivalent solid beams with the same bending stiffness as the real stacks (see table II) instead of complex structures.
Finally, high enough via density is required in order to avoid via detachment from the metal layers due to out-of-plane forces. Via detachment has been observed in our preliminary test designs when very low via density was used, but it has not been observed for any of the presented via designs, which were used in several other MEMS structures.

\section{K. Temperature and Time Effects on Curvature}

Thermal annealing can modify the curvature of thin-film structures [40, 41]. Also, temperature effects on curvature of released BEOL layers were already identified in [42]. Here, they have been investigated by applying different thermal profiles to 18 samples $(3 \mathrm{~cm} \mathrm{x} 3 \mathrm{~cm})$ that resulted from cleaving LF wafer 2 from run 4, lot 1 (see table V). Each thermal profile presented a different maximum temperature $\left(150^{\circ} \mathrm{C}-400{ }^{\circ} \mathrm{C}\right)$ and duration $(1 \mathrm{~min}, 15 \mathrm{~min}$ or $60 \mathrm{~min})$. The rising ramp was $1{ }^{\circ} \mathrm{Cs}^{-1}$, whereas the measured cooling down ramp was $0.3{ }^{\circ} \mathrm{C} \mathrm{s}^{-1}$, approximately. The curvature of 4 test cantilevers on each sample was measured after the thermal test, which was carried out in a nitrogen environment. Unfortunately, the sample corresponding to $400^{\circ} \mathrm{C}$ and $60 \mathrm{~min}$ was damaged during manipulation, so the associated data point is missing. The obtained mean curvature for each sample is plotted in figure 12. Note that the initial curvature of each sample is not exactly the same due to the curvature nonuniformity over the wafer.

Results showed that the qualitative response to temperature of single metals was very similar to those of stacks. For both of them the curvature became more positive the higher the temperature and the larger the time duration. However, the final curvature of the stacks was 10-20 times lower than the curvature of single metals. Also, it seems there is a timetemperature threshold above which the curvature changes quite abruptly: transitions from $250{ }^{\circ} \mathrm{C}$ to $350{ }^{\circ} \mathrm{C}$ exhibited the larger curvature variations. Also, time effects were most noticeable at $300{ }^{\circ} \mathrm{C}$, where the transition from 1 minute to 15 minutes leads to important curvature variations. At higher temperatures, time effects were much less important and the new more positive 


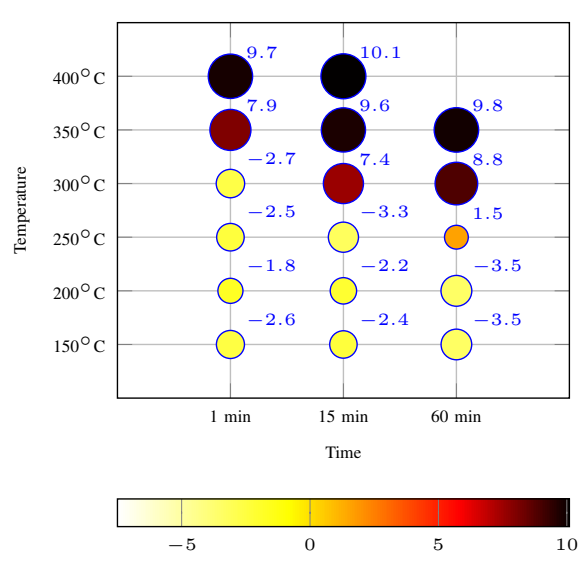

(a) Curvature $\left(\mathrm{mm}^{-1}\right)$ of M3

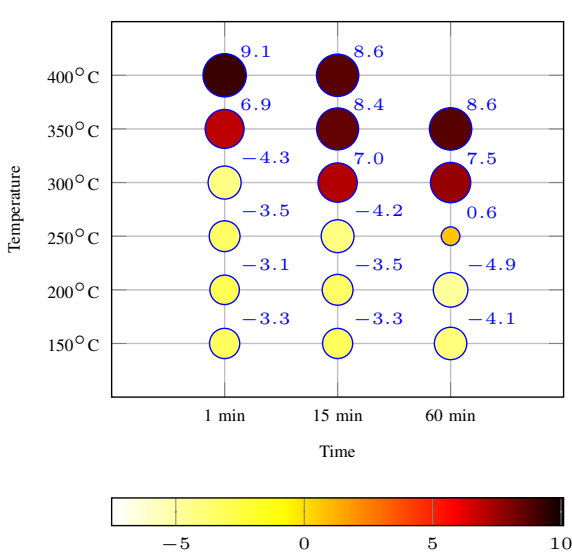

(b) Curvature $\left(\mathrm{mm}^{-1}\right)$ of M4

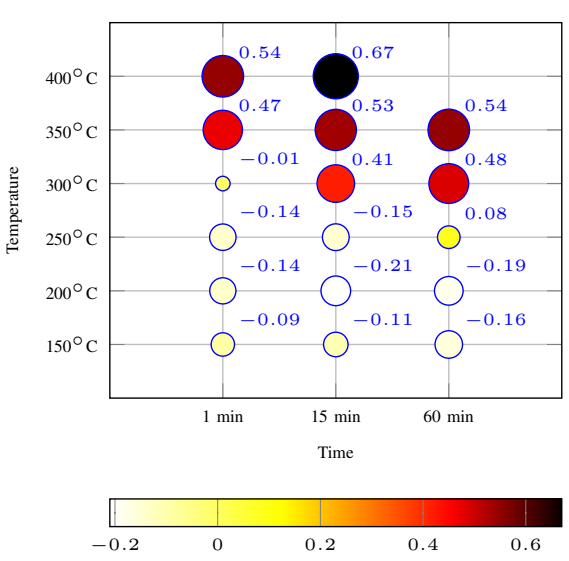

(c) Curvature $\left(\mathrm{mm}^{-1}\right)$ of M345

Figure 12: Effect of temperature and time on the curvature of cantilevers after cool down: Wafer 2, Lot 1, Run 4 from LFoundry. Size of data points indicate absolute value of the curvature, whereas color indicates the signed value as described by the scale below the graph.

curvature was more stable with time.

The observed time-temperature threshold cannot be attributed to the $\mathrm{W}$ vias of the stacks given that it took place in both single metals (which have no $\mathrm{W}$ vias) and stacks. Also, it is difficult to justify that such a large increase in the curvature of the stacks may be caused by sublimation of residues on the surface of the cantilevers. On the other hand, thermal stresses develop with increasing temperature due to the unequal thermal expansion coefficient of the layers that form the cantilevers ( $\mathrm{Al}, \mathrm{Ti}$ and $\mathrm{TiN}$ ). If the yield stress were surpassed plastic deformation would take place, leaving the cantilevers deformed after cooling down. As a matter of fact, permanent deformation of BEOL CMOS-MEMS cantilevers after being subjected to high temperatures has been attributed [24] to plastic deformation due to aluminum yield strength reduction at high temperatures [26, 43-45]. However, in order to be able to account for the observed time-dependence in our experiments, yield strength time-dependence [45, 46] cannot be neglected as in the mentioned study. In addition, we think that thermal creep mechanisms, highly dependent on stress and temperature levels $[47,48]$, should also play an important role in the observed permanent deformations, especially for the longer times. In fact, aluminum and its alloys are prone to creep at relative low temperatures given their low melting point ( $660{ }^{\circ} \mathrm{C}$ for pure aluminum and lower for alloys)[46]. Once the cantilevers deform enough to relieve the stress significantly, creep rate should slow down greatly given its very important dependence on stress levels. This could qualitatively explain the more stable, but still time-dependent, new more positive curvatures at $350{ }^{\circ} \mathrm{C}$ and $400{ }^{\circ} \mathrm{C}$.

In any case, the presented data can be used for predicting the behavior of BEOL CMOS-MEMS structures after being subjected to high temperatures, like when going through some of the typical packaging steps of MEMS devices, many of which involve important temperature excursions. For example, from these data we could have deduced that the baking step needed after the vHF release [17] should not induce noticeable curvature variations given its maximum temperature (around $200-250^{\circ} \mathrm{C}$ ) and time duration (around 30 seconds). As a matter of fact, this agrees with the results shown in figure 9 and presented in section V-E.

Data also shows that, theoretically, curvature reduction may be achieved in some cases by applying the right temperature profile to the BEOL structures. For example, $1 \mathrm{~min}$ at $300{ }^{\circ} \mathrm{C}$ reduced the curvature of $\mathrm{M} 345$ from $-0.09 \mathrm{~mm}^{-1}$ to $-0.01 \mathrm{~mm}^{-1}$ (see figure $12 \mathrm{c}$ ) or $60 \mathrm{~min}$ at $250{ }^{\circ} \mathrm{C}$ reduced the M4 curvature from $-4.1 \mathrm{~mm}^{-1}$ to $0.6 \mathrm{~mm}^{-1}$ (see figure $12 \mathrm{~b}$ ).

\section{CONCLUSIONS}

Curvature characterization of structural layers is essential for succesful development of MEMS products. Related literature for metal structures of CMOS-MEMS processes is currently very limited. A methodology for measuring cantilever curvature was presented, and its limitations analyzed. In particular, curvature measurement accuracy was discussed and modeled succesfully with an equation applicable for general cantilever curvature measurement techniques. For our setup, $\pm 0.0021 \mathrm{~mm}^{-1}$ for $200 \mu \mathrm{m}$-long cantilevers was achieved.

Extensive curvature measurement of CMOS-MEMS BEOL released cantilevers was carried out on $0.18 \mu \mathrm{m}$ wafers from two different CMOS foundries. Test cantilevers composed of several stacked metal layers were included in all the tested runs, and analytical equations that describe their bending behavior as a function of the $\mathrm{W}$ via design that join the layers were presented. Remarkably, the $\mathrm{W}$ via design most used in this work (standard rectangular arrays of $\mathrm{W}$ vias) does not contribute to the bending stiffness of the stack or its curvature. However, important curvature modification of the stacks by employing other $\mathrm{W}$ via designs was demonstrated.

Curvature of single metal layers was typically in the order of a few $\mathrm{mm}^{-1}$ and its WIW variability between 0.5 and $1.0 \mathrm{~mm}^{-1}$. Unfortunately, this imposes serious restrictions to design competitive MEMS that may be circumvented in many cases by stacking several layers. Stacking 4 identical metal layers reduced not only the mean curvature between 1 and 2 orders of magnitude with respect to single metals but also, and more importantly, its variability between 20 and 40 times. The curvature reduction was highly correlated with 
the number of stacked layers and was modeled analytically. Curvature reductions varied from case to case, highlighting the importance of stress mismatch between single layers. In addition, gap variations, and consequently CMP uniformity, were shown to be an important factor in stack curvature variability control. WIW variability usually followed a radial distribution although other distributions were also observed for stacks of metals.

Process variations like processing or not the FEOL, backgrinding, the baking step, release machine vendor or release etch duration showed no noticeable curvature effects compared to other sources of variability.

Finally, temperature was found to induce a severe curvature increase of single metals and stacks when a certain time-dependent temperature threshold was surpassed, which approximately ranged from $300-350{ }^{\circ} \mathrm{C}$ during 1 minute to $250^{\circ} \mathrm{C}$ during 1 hour.

The present work will aid MEMS designers in assessing whether the analyzed CMOS-MEMS processes are suitable for a given MEMS application. Also, the results and the characterization methodology presented herein are useful for MEMS designers who work with a similar manufacturing approach in order to shorten the development time and reduce the number of design iteration loops.

\section{ACKNOWLEDGMENT}

The authors would like to thank Sandra Aguilar from Baolab Microsystems for assistance during the measurements. This work was supported by Baolab Microsystems and by the Spanish Ministry of Economy and Competitivity under Project TEC2015-67278-R and the European Social Fund (ESF).

\section{REFERENCES}

[1] H. Baltes, O. Brand, A. Hierlemann, D. Lange, and C. Hagleitner, "CMOS MEMS - present and future," in Micro Electro Mechanical Systems, 2002. The Fifteenth IEEE International Conference on, 2002, pp. $459-466$.

[2] D. Fernández, J. Ricart, and J. Madrenas, "Experiments on the release of CMOS-micromachined metal layers," Journal of Sensors, vol. 2010, 2010.

[3] M.-H. Tsai, Y.-C. Liu, K.-C. Liang, and W. Fang, "Monolithic CMOS-MEMS Pure Oxide Tri-Axis Accelerometers for Temperature Stabilization and Performance Enhancement," Journal of Microelectromechanical Systems, vol. 24, no. 6, pp. 1916-1927, 2015.

[4] B. Bhushan, Tribology Issues and Opportunities in MEMS: Proceedings of the NSF/AFOSR/ASME Workshop on Tribology Issues and Opportunities in MEMS held in Columbus, Ohio, USA, 9-11 November 1997. Springer Science \& Business Media, 2012.

[5] W.-C. Chen, W. Fang, and S.-S. Li, "A generalized CMOS-MEMS platform for micromechanical resonators monolithically integrated with circuits," Journal of Micromechanics and Microengineering, vol. 21, no. 6, p. 065012, 2011.

[6] G. K. Fedder, "Integrated MEMS in conventional CMOS," in Tribology Issues and Opportunities in MEMS. Springer, 1998, pp. 17-29.

[7] H. Qu, "CMOS MEMS Fabrication Technologies and Devices," Micromachines, vol. 7, no. 1, p. 14, 2016.

[8] G. Zhang, H. Xie, L. E. de Rosset, and G. K. Fedder, "A lateral capacitive CMOS accelerometer with structural curl compensation," in Technical Digest. IEEE International MEMS 99 Conference. Twelfth IEEE International Conference on Micro Electro Mechanical Systems (Cat. No.99CH36291), Jan 1999, pp. 606-611.

[9] G. K. Fedder and R. D. S. Blanton, "Characterization and reliability of CMOS microstructures," pp. 132-139, 1999.

[10] W. Fang and J. A. Wickert, "Determining mean and gradient residual stresses in thin films using micromachined cantilevers," Journal of
Micromechanics and Microengineering, vol. 6, no. 3, pp. 301-309, 1996.

[11] S. Iyer, H. Lakdawala, G. K. Fedder, and T. Mukherjee, "Macromodeling Temperature-Dependent Curl in CMOS Micromachined Beams," in MSM 2001, pp. 19-21, 2001.

[12] National Institute of Standards and Technology, "Standard Reference Database 166," http://pml.nist.gov/test-structures/MEMSCalculator.htm, [Online; accessed 01-Dec-2016].

[13] C.-L. Cheng, M.-H. Tsai, and W. Fang, "Determining the thermal expansion coefficient of thin films for a CMOS MEMS process using test cantilevers," Journal of Micromechanics and Microengineering, vol. 25, no. 2, p. $025014,2015$.

[14] H. Lakdawala and G. K. Fedder, "Analysis of temperature-dependent residual stress gradients in CMOS micromachined structures," in Transducers, vol. 99, 1999, pp. 526-529.

[15] T.-H. Yen, M.-H. Tsai, C.-I. Chang, Y.-C. Liu, S.-S. Li, R. Chen, J.C. Chiou, and W. Fang, "Improvement of CMOS-MEMS accelerometer using the symmetric layers stacking design," in Sensors, 2011 IEEE. IEEE, 2011, pp. 145-148.

[16] C.-H. Hsueh, "Modeling of elastic deformation of multilayers due to residual stresses and external bending," Journal of Applied physics, vol. 91, no. 12, pp. 9652-9656, 2002.

[17] J. Valle, D. Fernández, and J. Madrenas, "Experimental Analysis of Vapor HF Etch Rate and Its Wafer Level Uniformity on a CMOS-MEMS Process," Journal of Microelectromechanical Systems, vol. 25, no. 2, pp. 401-412, 2016.

[18] J. Montanya i Silvestre, J. Valle Fraga, L. Barrachina Saralegui, and D. Fernandez Martinez, "MEMS devices and sensors in standard CMOS processing," in Solid-State Sensors, Actuators and Microsystems, Transducers Eurosensors XXVII, June 2013, pp. 713-717.

[19] S. Timoshenko, Strength of materials. Krieger Pub. Co, 1983.

[20] Lyncéetec, http://www.lynceetec.com/reflection-dhm/, [Online; accessed 04-Oct-2016].

[21] T.-S. Park, M. Dao, S. Suresh, A. Rosakis, D. Pantuso, and S. Shankar, "Some practical issues of curvature and thermal stress in realistic multilevel metal interconnect structures," Journal of Electronic Materials, vol. 37 , no. 6 , pp. 777-791, 2008

[22] M. Bakri-Kassem, S. Fouladi, and R. R. Mansour, "Novel high-Q MEMS curled-plate variable capacitors fabricated in 0.35- $\mu \mathrm{m}$ CMOS technology," IEEE Transactions on Microwave Theory and Techniques, vol. 56, no. 2, pp. 530-541, 2008.

[23] H. Xie and G. K. Fedder, "Vertical comb-finger capacitive actuation and sensing for CMOS-MEMS," Sensors and Actuators A: Physical, vol. 95 , no. 2, pp. 212-221, 2002.

[24] F. Y. Kuo, C. S. Chang, Y. S. Liu, K. A. Wen, and L. S. Fan, "Temperature-dependent yield effects on composite beams used in CMOS MEMS," Journal of Micromechanics and Microengineering, vol. 23, no. 3, p. 035023, 2013.

[25] J. Hasselbach, K. Lestage, and A. Stamper, "Oxide mems beam," Patent, May 31, 2012, uS Patent App. 12/955,220.

[26] P. A. Flinn, D. S. Gardner, and W. D. Nix, "Measurement and interpretation of stress in aluminum-based metallization as a function of thermal history," IEEE Transactions on electron devices, vol. 34, no. 3, pp. 689699, 1987.

[27] V. A. Aksyuk, F. Pardo, and D. J. Bishop, "Stress-induced curvature engineering in surface-micromachined devices," in Design, Test, and Microfabrication of MEMS/MOEMS. International Society for Optics and Photonics, 1999, pp. 984-993.

[28] T. C. Hodge, S. A. Bidstrup-Allen, and P. A. Kohl, "Stresses in thin film metallization," IEEE Transactions on Components, Packaging, and Manufacturing Technology: Part A, vol. 20, no. 2, pp. 241-250, 1997.

[29] W. Fang and C.-Y. Lo, "On the thermal expansion coefficients of thin films," Sensors and Actuators A: Physical, vol. 84, no. 3, pp. 310-314, 2000.

[30] M. Birkholz, K.-E. Ehwald, P. Kulse, J. Drews, M. Fröhlich, U. Haak, M. Kaynak, E. Matthus, K. Schulz, and D. Wolansky, "Ultrathin TiN Membranes as a Technology Platform for CMOS-Integrated MEMS and BioMEMS Devices," Advanced Functional Materials, vol. 21, no. 9, pp. 1652-1656, 2011.

[31] X. Hiao, Introduction to semiconductor manufacturing technology. Prentice Hall, 2000.

[32] M. Bhushan and M. B. Ketchen, Microelectronic test structures for CMOS technology. Springer Science \& Business Media, 2011.

[33] D. Guan, A. Bruccoleri, R. Heilmann, and M. Schattenburg, "Stress control of plasma enhanced chemical vapor deposited silicon oxide film from tetraethoxysilane," Journal of Micromechanics and Microengineering, vol. 24, no. 2, p. 027001, 2013. 
[34] I. Blech and U. Cohen, "Effects of humidity on stress in thin silicon dioxide films," Journal of Applied Physics, vol. 53, no. 6, pp. 4202 4207, 1982.

[35] J. Bühler, F.-P. Steiner, and H. Baltes, "Silicon dioxide sacrificial layer etching in surface micromachining," Journal of Micromechanics and Microengineering, vol. 7, no. 1, pp. R1-R13, 1997.

[36] A. Witvrouw, B. D. Bois, P. De Moor, A. Verbist, C. A. Van Hoof, H. Bender, and C. Baert, "Comparison between wet HF etching and vapor HF etching for sacrificial oxide removal," in Proc. SPIE, vol. 4174, no. 1. Santa Clara, CA, USA: SPIE, Aug. 2000, pp. 130-141.

[37] "Lab Manual of Marvell Nanofabrication Laboratory for the Primaxx tool," https://nanolab.berkeley.edu/labmanual/chap7/7.07primaxx.pdf, [Online; accessed 22-Nov-2016].

[38] W. C. Young and R. G. Budynas, Roark's formulas for stress and strain. McGraw-Hill New York, 2002, vol. 7.

[39] J. C. Marshall, D. L. Herman, P. T. Vernier, D. L. DeVoe, and M. Gaitan, "Young's modulus measurements in standard IC CMOS processes using MEMS test structures," IEEE Electron Device Letters, vol. 28, no. 11 pp. 960-963, 2007.

[40] X. Zhang, T.-Y. Zhang, M. Wong, and Y. Zohar, "Rapid thermal annealing of polysilicon thin films," Journal of Microelectromechanical Systems, vol. 7, no. 4, pp. 356-364, Dec 1998.

[41] S. Huang, B. Li, and X. Zhang, "Elimination of stress-induced curvature in microcantilever infrared focal plane arrays," Sensors and Actuators A: Physical, vol. 130, pp. 331-339, 2006.

[42] M.-A. Eyoum, N. Hoivik, C. Jahnes, J. Cotte, and X.-H. Liu, "Analysis and modeling of curvature in copper-based MEMS structures fabricated using CMOS interconnect technology," in The 13th International Conference on Solid-State Sensors, Actuators and Microsystems, 2005. Digest of Technical Papers. TRANSDUCERS'05., vol. 1. IEEE, 2005, pp. 764-767.

[43] R. Molina, P. Amalberto, and M. Rosso, "Mechanical characterization of aluminium alloys for high temperature applications Part1: Al-Si-Cu alloys," Metallurgical Science and Tecnology, vol. 29, no. 1, 2013.

[44] - "Mechanical characterization of aluminium alloys for high temperature applications Part 2: Al-Cu, Al-Mg alloys," Metallurgical Science and Tecnology, vol. 29, no. 2, 2013.

[45] R. C. Rice, Metallic Materials Properties Development and Standardization (MMPDS): Chapters 1-4. National Technical Information Service, 2003, vol. 1.

[46] P. T. Summers, Y. Chen, C. M. Rippe, B. Allen, A. P. Mouritz, S. W. Case, and B. Y. Lattimer, "Overview of aluminum alloy mechanical properties during and after fires," Fire Science Reviews, vol. 4, no. 1, p. 3, 2015.

[47] S. Spigarelli, "Creep of aluminum and aluminum alloys," TALAT Lecture, vol. 1253, 1999.

[48] W. Fang, H.-C. Tsai, and C.-Y. Lo, "Determining thermal expansion coefficients of thin films using micromachined cantilevers," Sensors and Actuators A: Physical, vol. 77, no. 1, pp. 21-27, 1999.

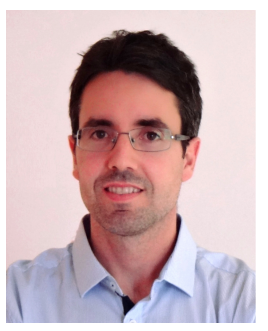

Juan Valle was born in Lugo, Spain, in 1977. He received the M.Sc. degree in Physics in 2000 and the M.Sc. degree in Industrial Engineering in 2002 from Universidad Alfonso X El Sabio (UAX), Madrid, Spain. He received the International Master's in Theoretical and Practical Application of Finite Element Method and CAE Simulation in 2004 from Universidad Nacional a Distancia (UNED), Madrid, Spain.

From 2001 to 2002, he worked as a Microsystems (MEMS) and Nanotechnology consultant for the INTA (National Institute for Aerospace Technology). He specialized on Multiphysics simulations before joining Delphi Diesel Systems in 2004 as an Analyst Engineer, and joined Baolab Microsystems in 2005 where he researched on the fields of MEMS sensors and micromanufacturing processes for 9 years, where he filled 10 patent applications on related fields. Juan Valle devised design techniques applicable for the MEMS fabrication inside the CMOS BEOL. Using these techniques he is developing the first CMOSMEMS 3 axis magnetometer aimed at mass production. Currently he is pursuing his Ph.D. in Electronic Engineering at Universitat Politècnica de Catalunya (UPC), Barcelona, Spain.

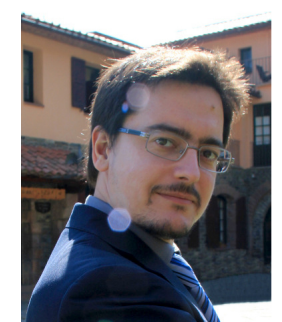

Daniel Fernández was born in Barcelona, Spain, in 1979. He received the M.Sc. degree in telecommunications engineering, Ph.D. degree (cum laude), and M.B.A. degree from Universitat Politècnica de Catalunya (UPC), Barcelona, Spain, in 2004, 2008 and 2009, respectively.

From 2008 to 2010 he worked as a Postdoctoral Researcher at the Electronic Engineering Department, UPC in the fields of CMOS surface micromachining, circuits and control architectures for MEMS sensors and actuators, translinear circuits for analog signal processing and digital implementations of power converters. From 2010 to 2014 he worked as Principal ASIC Engineer at Baolab Microsystems developing circuits and architectures for CMOS MEMS/NEMS-based products, and as ASIC Design Engineer Contractor for the European Space Agency designing radiation-hardened integrated-circuits and interface blocks for space exploration in interplanetary missions. Currently he is Chief Technology and Science Officer at Nanusens, Bellaterra, Spain, where he works towards the development of innovative circuits and architectures for MEMS sensors signalconditioning.

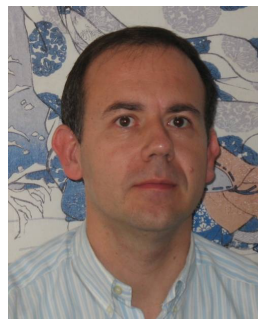

Jordi Madrenas Jordi Madrenas (Telecom. Eng. '86, Ph.D. '91, UPC, Barcelona, Catalunya, Spain) is currently Associate Professor at the Department of Electronic Engineering, Technical University of Catalunya (UPC BarcelonaTech). He has participated in 5 European projects and coordinated 5 Spanish national research projects. Also, he has coordinated several contracts with companies. At present, he coordinates a national project on MEMSon-chip and microsensor bio-inspired signal processing. He has co-authored more than 120 scientific journal and international conference papers, two books and 5 book chapters. Between 2000 and 2003 he was Vice-Dean of Studies of the Telecommunication Engineering School of Barcelona, UPC. His current research interests include analog, mixed-signal and digital VLSI and FPGA design, CMOS-MEMS design and conditioning, ultra low-power design, bioinspired/neuromorphic system implementation and rad-hard mixed-signal circuits.

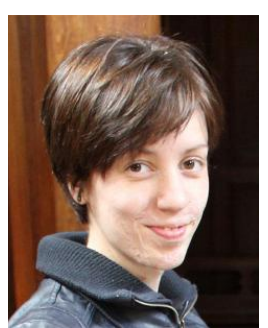

Laura Barrachina Laura Barrachina was born in Pamplona, Spain, in 1981. She received the M.Sc. degree in Physics in 2004 from Universidad de Zaragoza, and Master of Advanced Studies in Microelectronics from Universidad de Barcelona, Spain.

She joined the National Microelectronics Center in Barcelona for 2 years, where she worked developing a methodology to design a MEMS by modelling in VHDL-AMS a 3-axis accelerometer. From 2006 to 2014, she worked at Baolab Microsystems as a Test and Measurement engineer, performing automated measurements at wafer level, specializing in MEMS sensor characterization. Since 2014, she is working at Owlstone, Cambridge, as a System Engineer in chemical detectors based on field asymmetric ion mobility spectrometry. 